\title{
Dysregulations of MicroRNA and Gene Expression in Chronic Venous Disease
}

\author{
Daniel P. Zalewski ${ }^{1,+}{ }^{\dagger}$ Karol P. Ruszel ${ }^{2,+}$, Andrzej Stępniewski ${ }^{3}$, Dariusz Gałkowski ${ }^{4}$, \\ Jacek Bogucki ${ }^{2}{ }^{\circledR}$, tukasz Komsta ${ }^{5}$, Przemysław Kołodziej ${ }^{1}{ }^{10}$, Paulina Chmiel ${ }^{1}$, \\ Tomasz Zubilewicz ${ }^{6}$, Marcin Feldo ${ }^{6, \ddagger}$, Janusz Kocki ${ }^{2, \ddagger}$ a and Anna Bogucka-Kocka ${ }^{1, *, \ddagger}$
}

1 Chair and Department of Biology and Genetics, Medical University of Lublin, 4a Chodźki St., 20-093 Lublin, Poland; daniel.piotr.zalewski@gmail.com (D.P.Z.); przemyslaw.kolodziej@umlub.pl (P.K.); pachmiel13@gmail.com (P.C.)

2 Chair of Medical Genetics, Department of Clinical Genetics, Medical University of Lublin, 11 Radziwiłłowska St., 20-080 Lublin, Poland; karol.ruszel@umlub.pl (K.P.R.); jacek.bogucki@umlub.pl (J.B.); janusz.kocki@umlub.pl (J.K.)

3 Ecotech Complex Analytical and Programme Centre for Advanced Environmentally Friendly Technologies, University of Marie Curie-Skłodowska, 39 Głęboka St., 20-612 Lublin, Poland; andrzej.stepniewski@umcs.pl Department of Pathology and Laboratory Medicine, Rutgers-Robert Wood Johnson Medical School, One Robert Wood Johnson Place, New Brunswick, NJ 08903-0019, USA; galkowd@fastmail.fm

5 Chair and Department of Medicinal Chemistry, Medical University of Lublin, 4 Jaczewskiego St., 20-090 Lublin, Poland; lukasz.komsta@umlub.pl

6 Chair and Department of Vascular Surgery and Angiology, Medical University of Lublin, 11 Staszica St., 20-081 Lublin, Poland; tomasz.zubilewicz@umlub.pl (T.Z.); martinf@interia.pl (M.F.)

* Correspondence: anna.kocka@umlub.pl; Tel.: +48-81-448-7232

+ D.P.Z. and K.P.R. shared first authorship.

$\ddagger \quad$ M.F., J.K. and A.B.-K shared senior authorship.

Received: 26 March 2020; Accepted: 21 April 2020; Published: 25 April 2020

Abstract: Chronic venous disease (CVD) is a vascular disease of lower limbs with high prevalence worldwide. Pathologic features include varicose veins, venous valves dysfunction and skin ulceration resulting from dysfunction of cell proliferation, apoptosis and angiogenesis. These processes are partly regulated by microRNA (miRNA)-dependent modulation of gene expression, pointing to miRNA as a potentially important target in diagnosis and therapy of CVD progression. The aim of the study was to analyze alterations of miRNA and gene expression in CVD, as well as to identify miRNA-mediated changes in gene expression and their potential link to CVD development. Using next generation sequencing, miRNA and gene expression profiles in peripheral blood mononuclear cells of subjects with CVD in relation to healthy controls were studied. Thirty-one miRNAs and 62 genes were recognized as potential biomarkers of CVD using DESeq2, Uninformative Variable Elimination by Partial Least Squares (UVE-PLS) and ROC (Receiver Operating Characteristics) methods. Regulatory interactions between potential biomarker miRNAs and genes were projected. Functional analysis of microRNA-regulated genes revealed terms closely related to cardiovascular diseases and risk factors. The study shed new light on miRNA-dependent regulatory mechanisms involved in the pathology of CVD. MicroRNAs and genes proposed as CVD biomarkers may be used to develop new diagnostic and therapeutic methods.

Keywords: chronic venous disease; CVD; varicose veins; miRNA; microRNA; gene; expression; next generation sequencing; biomarker 


\section{Introduction}

Chronic Venous Disease (CVD) is a common vascular disease of the lower limbs. Estimates of the worldwide prevalence of symptomatic CVD range as high as $60 \%[1,2]$. The common risk factors include age, obesity, smoking, low physical activity, periods of prolonged standing or sitting and positive family history [2]. CVD is defined as a syndrome of chronic morphological and functional abnormalities of the venous system, manifested either by symptoms (including tingling, aching, burning, pain, muscle cramps, swelling, sensations of throbbing or heaviness, itching, restless legs, leg tiredness and fatigue) or clinical signs prompting the need for investigation and medical care [3]. The disease encompasses a wide spectrum of clinical presentations such as telangiectasia, varicose veins, leg edema, skin changes and ulcers, as included in the Clinical, Etiology, Anatomic, Pathophysiology (CEAP) classification [4-6]. Chronic Venous Insufficiency (CVI) is a term reserved for advanced CVD, which is applied to functional abnormalities of the venous system producing edema, skin changes, or venous ulcers (C3-C6 in CEAP classification) [3].

CVD is caused by hemodynamic disturbances in veins of lower limbs, presenting as venous occlusion, venous valve incompetency and calf muscle pump dysfunction, which lead to venous hypertension and reflux [5,7-9]. In the majority of cases, the great and small saphenous veins are involved [8]. The skin manifestations, such as edema, dermatitis, lipodermatosclerosis and ulceration, are the consequence of chronic volume overload and hypertension in subcutaneous veins, as well as inflammatory processes within skin tissues $[10,11]$. Imbalance between metalloproteinases and their inhibitors as well as accumulation of leukocytes in the tissues surrounding the venous vessels of the lower limbs under high pressure conditions is considered to be the basis for chronic inflammation and pathological changes in the skin tissue of patients with chronic venous insufficiency $[7,12,13]$.

A significant decrease in life quality, caused by pain, reduced mobility and leg ulcerations, affects patients with CVD [14,15].

The most recommended diagnostic procedures of CVD include physical examination and duplex ultrasound scanning, and the most effective treatment options are compression therapy and invasive interventions, complemented by pharmacotherapy $[16,17]$. New, more effective diagnostic and treatment strategies are needed and more profound understanding of pathology, particularly the interactions between molecular and cellular mechanisms, is essential for development of optimal treatment approaches.

MicroRNA (miRNA) has been the subject of many studies, greatly expanding the knowledge of their diversity and functions [18]. MiRNAs are approximately 18-25-nucleotides long, single-stranded RNAs involved in modulating gene expression pathways [19]. MiRNAs incorporated in protein complex exhibit gene expression regulating effect by binding to mRNA. The pairing effect of miRNA-mRNA interactions reduces gene expression predominantly by repression of translation, destabilization and cleavage of mRNA [20]. MiRNAs exert their effect as a switch and a fine-tuner of gene expression, providing a pleiotropic effect on protein pool in cells [21]. Alterations in genes involved in miRNA processing were found in various human cancers [22]. MiRNAs are intensively studied as potential means of novel diagnostic and treatment approaches $[23,24]$

A growing amount of evidence suggests a relevant role of miRNA in vascular cell functions, including cell differentiation, proliferation, migration, and apoptosis [25,26]. MiRNAs are involved in vascular diseases, exhibiting modulatory function of angiogenesis, endothelial cells dysfunction and response for ischemic events [27]. Numerous miRNAs are considered as potential markers of cardiovascular diseases (e.g., coronary artery disease, myocardial infarction, atherosclerosis, venous thromboembolism), exhibiting promising diagnostic, prognostic and therapeutic value [27-31].

Altered expression patterns of miRNAs and genes were demonstrated in vein specimens derived from patients with CVI and compared to healthy subjects [32,33]. Dysregulation of miRNA expression, reported in venous ulcers biopsies, has been associated with inhibition of wound healing [34]. The proposed role of miRNA in CVI susceptibility has also been reported [35]. Therefore, miRNAs 
could also be involved in the pathogenesis of CVD and may be relevant as potential diagnostic and therapeutic targets.

In our work, integrated miRNA and gene expression analysis was applied to find potential robust biomarkers of CVD and to show the impact of miRNA-regulated genes on pathological processes governing CVD development.

\section{Experimental Section}

\subsection{Study Participants Characteristics}

The study was performed in accordance with the Declaration of Helsinki and approved by the Ethics Committee at Medical University of Lublin (approval No. KE-0254/341/2015). Participants were recruited between February 2016 and May 2017. All subjects gave their informed consent for inclusion before they participated in the study. The CVD group consisted of 34 patients diagnosed and hospitalized only due to CVD, without any other diagnosed vascular diseases or comorbidities, in the Independent Public Clinical Hospital No. 1 in Lublin, Poland. The control group comprised of 19 healthy volunteers without any visible CVD characteristics and lack of comorbidities during examination. Detailed characteristics of included participants are presented in Table 1.

Table 1. Characteristics of 34 patients with chronic venous disease (CVD) and 19 non-CVD controls included in the study.

\begin{tabular}{|c|c|c|c|}
\hline Characteristic & $\begin{array}{c}\text { CVD Population } \\
\quad(n=34)\end{array}$ & $\begin{array}{c}\text { Control Population } \\
\quad(n=19)\end{array}$ & $P$ \\
\hline Age & $\begin{array}{c}44.12 \pm 10.07^{1} \\
27-78^{2}\end{array}$ & $\begin{array}{c}36.58 \pm 9.97^{1} \\
24-55^{2}\end{array}$ & $8.387 \times 10^{-3}$ \\
\hline Body Mass Index & $\begin{array}{l}23.85 \pm 2.35^{1} \\
20.13-28.76^{2}\end{array}$ & $\begin{array}{c}23.12 \pm 3.93^{1} \\
19.33-32.6^{2}\end{array}$ & 0.117 \\
\hline Smoking: Current & $5(14.7 \%)$ & $0(0 \%)$ & \multirow{3}{*}{$1.296 \times 10^{-4}$} \\
\hline Smoking: Former & $13(38 \%)$ & $0(0 \%)$ & \\
\hline Smoking: Never & $16(47 \%)$ & $19(100 \%)$ & \\
\hline Sex: Male & $17(50 \%)$ & $9(47 \%)$ & \multirow{2}{*}{1} \\
\hline Sex: Female & $17(50 \%)$ & $10(53 \%)$ & \\
\hline \multicolumn{4}{|l|}{ Signs and symptoms } \\
\hline Pain & $7(20.6 \%)$ & NA & \\
\hline Ankle-brachial index & $\begin{array}{c}0.96 \pm 0.048^{1} \\
0.71-0.99^{2}\end{array}$ & NA & \\
\hline \multicolumn{4}{|l|}{ Extended anatomical classification } \\
\hline Great saphenous vein (above knee) & $23(67.7 \%)$ & NA & \\
\hline Great saphenous vein (below knee) & $7(20.6 \%)$ & NA & \\
\hline Small saphenous vein & $3(8.8 \%)$ & NA & \\
\hline Great and small saphenous vein & $1(2.9 \%)$ & NA & \\
\hline \multicolumn{4}{|l|}{ Medication } \\
\hline Micronized diosmin & $19(55.9 \%)$ & NA & \\
\hline $\begin{array}{l}\text { Preparation with vitaminum C, } \\
\text { hesperidin and Ruscus aculeatus extract }\end{array}$ & $10(29.4 \%)$ & NA & \\
\hline Both medications & $5(14.7 \%)$ & NA & \\
\hline
\end{tabular}


Inclusion and exclusion criteria were evaluated by a vascular surgeon. Included CVD patients were examined using tourniquet test, auscultation and duplex ultrasound scanning. Venous reflux lasting longer than $1 \mathrm{~s}$ was classified as pathological. Patients diagnosed with symptoms classified according to CEAP as varicose veins (C2) of superficial veins (As) with primary etiology (Ep) and reflux pathophysiology (Pr) were included. The exclusion criteria were previous vascular surgery of lower limbs, insufficiency of deep veins, acute and chronic inflammation of veins, lower extremities arterial disease, coronary artery disease, cerebrovascular disease, aneurismal disease, myocardial infarction, hypertension, stroke, diabetes mellitus type 2, and pregnancy.

Body Mass Index (BMI), pain symptoms, ankle-brachial index, smoking habits and applied medical treatment were also evaluated (Table 1).

The control group consisted of 19 healthy, nonsmoking volunteers (Table 1). Only subjects without blood flow disturbances and with normal morphology of veins in lower limbs, confirmed by physical examination and duplex ultrasound scanning, were included to the study as controls. Any symptoms, comorbidities and treatment of vascular diseases were indicated in the analysis of medical history of control subjects.

\subsection{Study Material Preparation}

Isolation of Peripheral Blood Mononuclear Cells (PBMCs) was performed from whole blood samples by density gradient centrifugation using Gradisol L reagent (Aqua-Med, Łódź, Poland). Small RNA fractions were isolated from PBMCs samples of 34 CVD patients and 19 controls using MirVana microRNA Isolation Kit (Ambion, Austin, TX, USA), according to the manufacturer's protocol. Total RNA was isolated from PBMCs of seven randomly selected CVD patients and seven randomly selected control subjects, using TRI Reagent Solution (Applied Biosystems, Foster, CA, USA) according to the manufacturer's protocol. For a more detailed description of study material isolation and assessment refer to [30].

\subsection{Libraries Preparation and Sequencing}

Small RNA libraries were prepared from 53 small RNA samples isolated from PBMCs of 34 CVD patients and 19 healthy controls. Technical limitations did not allow to perform transcriptome sequencing for all subjects included in the study, therefore transcriptome libraries were constructed from 14 total RNA samples isolated from randomly selected, representative subsets of PBMCs samples (seven from CVD patients and seven from healthy controls).

Small RNA and transcriptome libraries were prepared using Ion Total RNA-Seq Kit v2, Magnetic Bead Cleanup Module kit and barcoded with Ion Xpress RNA-Seq Barcode 01-16 Kit (all Life Technologies, Carlsbad, CA, USA), according to the manufacturer's protocol “Ion Total RNA-Seq Kit v2" revision B.0. Libraries were sequenced on Ion 540 Chips (Life Technologies) using Ion S5 XL System (ThermoFisher Scientific, Waltham, MA, USA). Small RNA and transcriptome raw sequencing data were aligned to 2792 human miRNAs from miRBase v21 (http://www.mirbase.org) and to 55,765 genes and splicing variants of hg19 human genome, respectively.

Detailed description of libraries preparation and sequencing procedures were included in our previous study [30].

\subsection{Statistical Analysis}

The differences between CVD and control groups were evaluated in terms of age and BMI using a two-sided Mann-Whitney $U$ test (wilcox.test function in $R$ ), and in terms of sex and smoking using Fisher exact test (fisher.test function in R).

Statistical analysis of miRNA and gene expression datasets was performed on biological replicates using $R$ environment (version 3.5.2, https://www.r-project.org) with proper packages. Differential expression analysis was carried out using DESeq2 package v1.18.1 (https://bioconductor. org/packages/release/bioc/html/DESeq2.html) [36]. MiRNAs and genes with $p$ value below 0.05 
after Benjamini-Hochberg false discovery rate correction were considered as statistically significant. Differential potential of miRNAs and genes was further confirmed by Uninformative Variable Elimination by Partial Least Squares (UVE-PLS) method [37] using the plsVarSel package v0.9.3 (https://cran.r-project.org/web/packages/plsVarSel/index.html) [38].

Venn diagrams, Heatmaps with Euclidean clustering and 3D Principal Component Analysis (PCA) plots were created using VennDiagram 1.6.20 (https://cran.r-project.org/web/packages/VennDiagram/ index.html) [39], pheatmap 1.0.10 (https://cran.r-project.org/web/packages/pheatmap/index.html) and scatterplot3d 0.3-41 (https://cran.r-project.org/web/packages/scatterplot3d/index.html) [40] packages, respectively. Correlation analysis was performed using Spearman rank correlation test implemented in the Hmisc package 4.4-0 (https://cran.r-project.org/web/packages/Hmisc/index.html).

A Receiver Operating Characteristics (ROC) analysis implemented in pROC package 1.12.1 [41] (https://cran.r-project.org/web/packages/pROC/index.html) was used to evaluate the predictive value of selected miRNAs and gene transcripts for CVD classification.

Deconvolution of gene expression was performed by "quanTIseq" [42] and "MCPcounter" [43] methods implemented to immunedeconv 2.0.0 package (https://rdrr.io/github/grst/ immunedeconv/) [44].

MultiMiR package 1.2.0 (https://bioconductor.org/packages/release/bioc/html/multiMiR.html) [45] was used to identify validated and predicted interactions between selected miRNAs and genes. Visualization of the regulatory network with interactions was performed using Cytoscape v3.5.1 software (https://cytoscape.org/) [46].

Functional analysis of networked genes was performed using Database for Annotation, Visualization and Integrated Discovery (DAVID) 6.8 tool (https://david.ncifcrf.gov/) [47,48]. Default whole genome of Homo sapiens was applied as a background. All the terms of Kyoto Encyclopedia of Genes and Genomes (KEGG), Reactome and Genetic Association Database (GAD) databases associated with analyzed genes were harvested. Enrichment analysis of functional terms was proceeded for Gene Ontology (GO) terms separately for up- and downregulated genes.

All statistical procedures applied to this study were previously described in detail in [30]. Statistical analysis and visualizations were performed according to $\mathrm{R}$ code available in published reference manuals of used packages.

The summarized methodology applied in our study is presented on Figure 1.

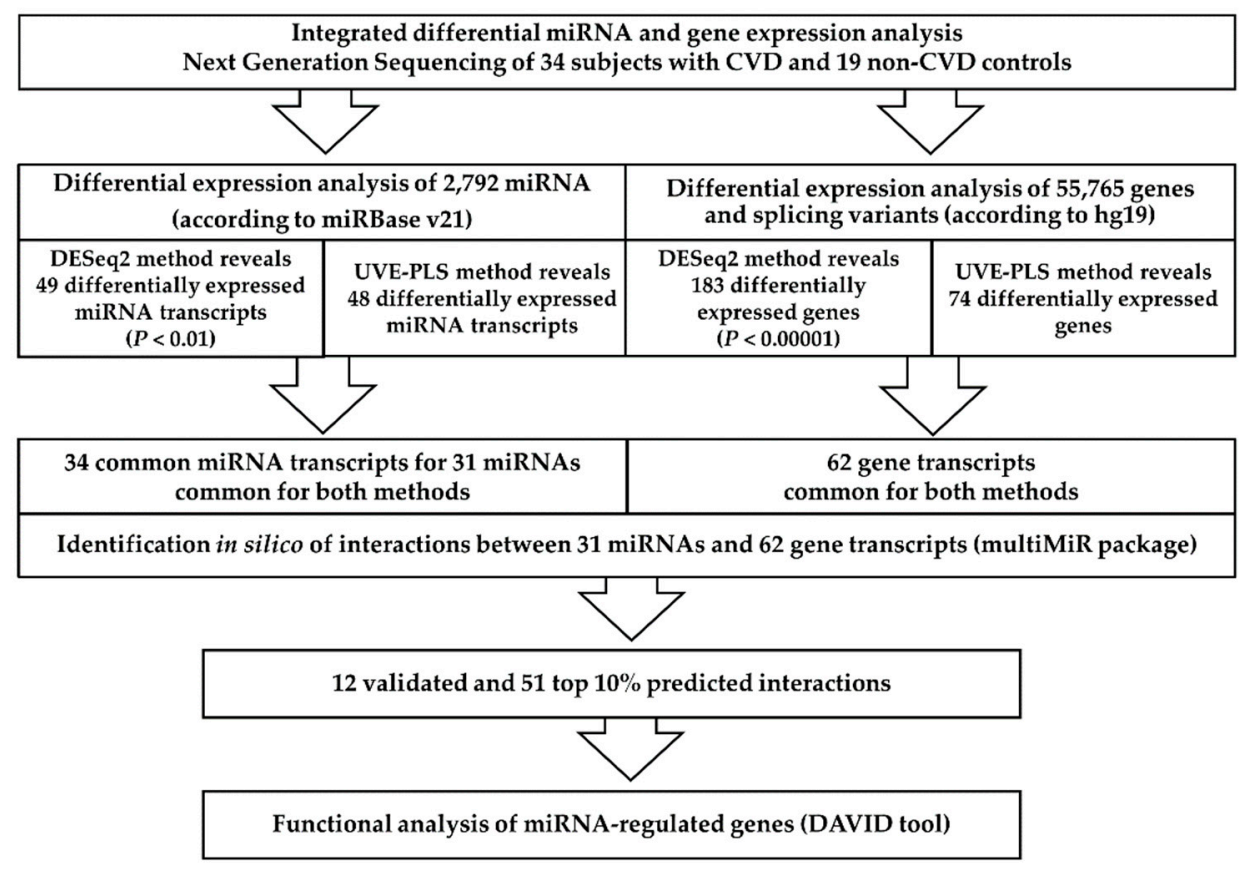

Figure 1. The scheme of the methodology applied in the study. CVD—chronic venous disease. 


\section{Results}

\subsection{Study Population Analysis}

Characteristics of 34 patients with CVD and 19 non-CVD controls are presented in Table 1. A statistically significant difference between these groups was observed in age $\left(p=8.387 \times 10^{-3}\right)$ and smoking history $\left(p=1.296 \times 10^{-4}\right)$, which probably results from inclusion of healthy CVD-negative non-smoking individuals in control group. No statistically significant differences between CVD and control groups concerning gender and BMI were found (Table 1, Figure S1).

\subsection{Primary Results}

Detailed description of small RNA samples and small RNA libraries as well as the results of primary analysis of small RNA libraries sequencing data are presented in Table S1. Detailed description of transcriptome libraries and results of primary analysis of transcriptome libraries sequencing data are presented in Table S2. Plots depicting sequencing data quality, including boxplot of Cook's distances, MA plot and histogram of $p$ values, regarding small RNA and transcriptome sequencing are presented in Figure S2 and Figure S3, respectively.

\subsection{Differential Expression Analysis of miRNA}

The comparison of miRNA expression levels between 34 CVD patients and 19 non-CVD controls was performed using DESeq2 and UVE-PLS methods and significantly dysregulated miRNAs selected by both methods were chosen.

DESeq2 analysis revealed 1034 differentially expressed miRNA transcripts in CVD subjects compared to controls. Ninety-six miRNA transcripts were differentially expressed with statistical significance $p<0.05$ (Table S3). DESeq2 method is characterized by relatively high sensitivity, therefore a set of 49 differentially expressed miRNA transcripts (for 41 miRNAs) of higher significance $(p<0.01)$ was selected to limit the number of potentially false positive results.

In order to optimally filter uninformative miRNAs, the UVE-PLS method was applied to miRNA expression data of 1034 differentially expressed miRNA transcripts in CVD subjects. UVE-PLS analysis returned 48 informative miRNA transcripts (Table S4). Figure S4 shows the arrangement of prediction error and PLS components as well as cross-validated predictions versus measured values.

The set of 49 differentially expressed miRNA transcripts identified by DESeq2 method (with $p<0.01$ ) and the set of 48 differentially expressed miRNA transcripts identified by UVE-PLS method were compared on the Venn diagram, revealing 34 miRNA transcripts common for both sets (Figure 2a). These 34 miRNA transcripts result in 31 miRNAs (22 upregulated and 9 downregulated), which constitute a proposed panel of potential miRNA biomarkers of CVD (Table 2). Differential expression of common 34 miRNA transcripts in CVD and control group is visualized on 3D PCA plot and in heatmap with Euclidean clustering (Figure 2b,c, respectively). 
a

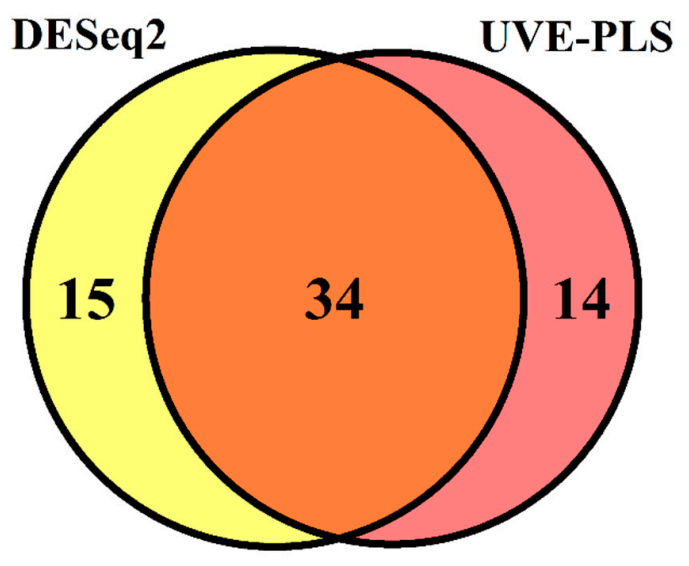

C

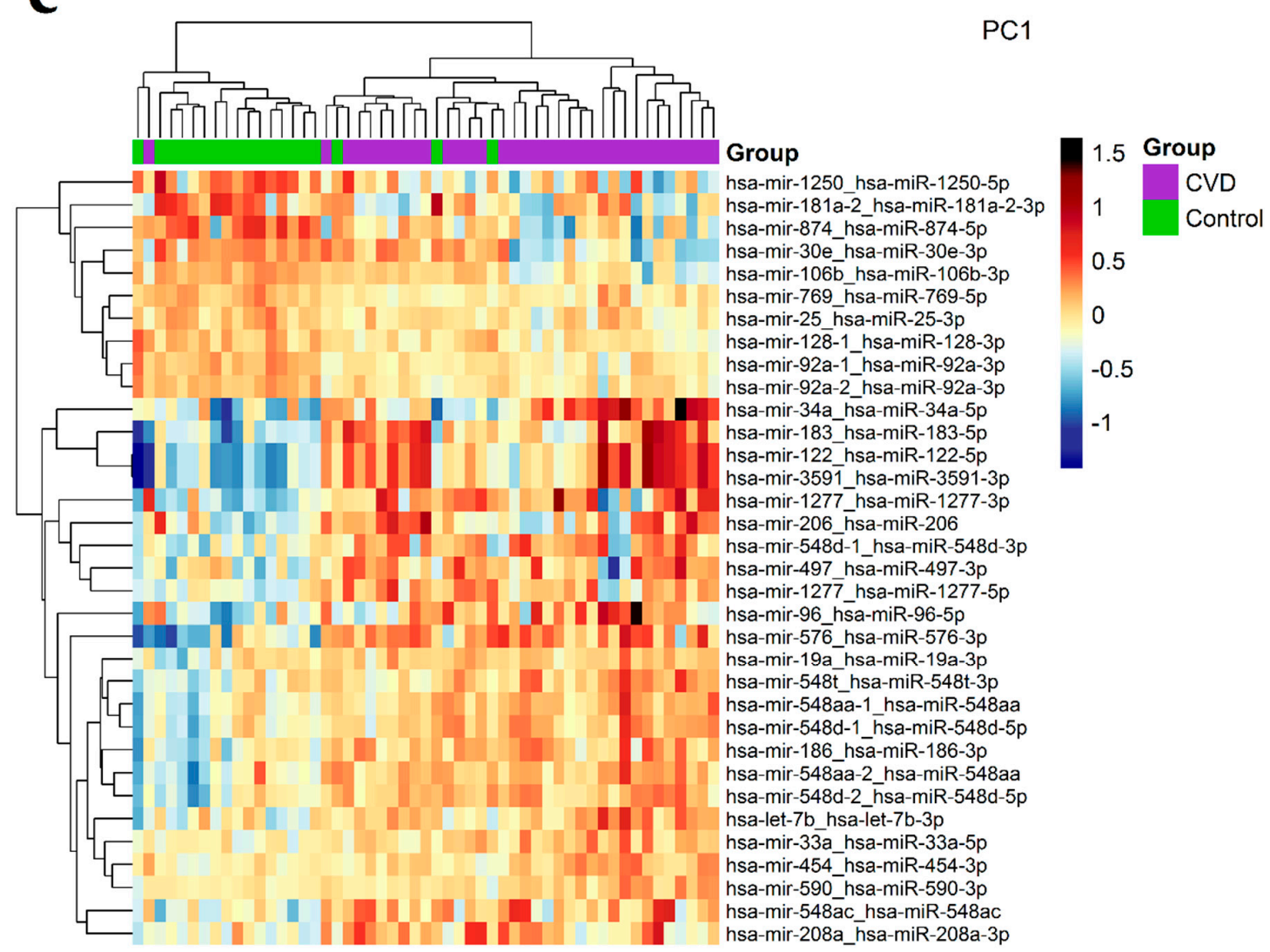

b

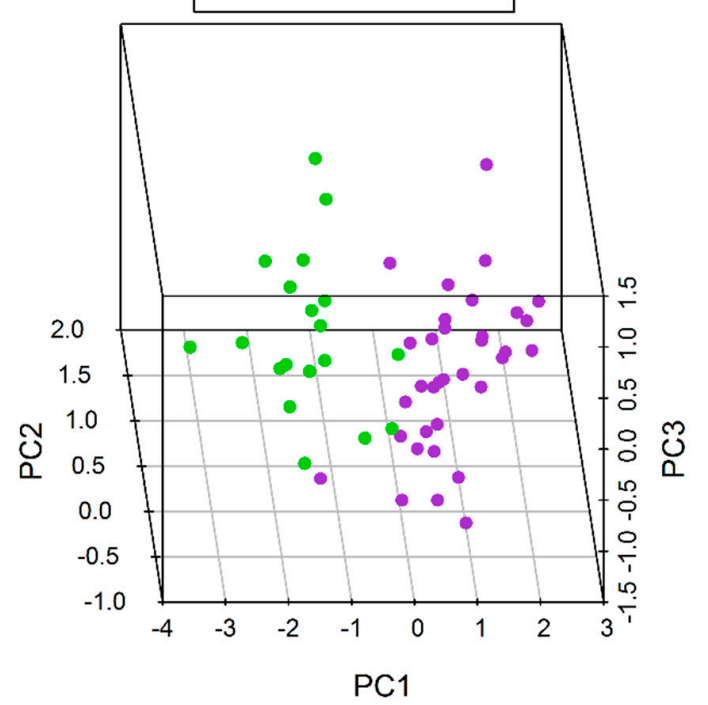

Figure 2. Results of differential expression analysis of miRNA in group of 34 patients with chronic venous disease (CVD) vs. 19 healthy controls (Control). (a) Venn diagram presenting comparison of two sets of miRNA transcripts: the set of 49 miRNA transcripts resulted from DESeq2 analysis with $p<0.01$ and the set of 48 informative miRNA transcripts resulted from Uninformative Variable Elimination by Partial Least Squares (UVE-PLS) analysis. Thirty-four miRNA transcripts were common for both sets. 3D Principal Component Analysis (PCA) plot (b) and heatmap with Euclidean clustering (c) show differential expression of common 34 miRNA transcripts in CVD and control groups. 
Table 2. Set of 34 differentially expressed miRNA transcripts resulted from DESeq2 analysis with $p<0.01$ with statistical significance confirmed by UVE-PLS analysis in 34 patients with chronic venous disease compared to 19 controls.

\begin{tabular}{|c|c|c|c|c|c|c|}
\hline No. & miRNA Transcript & $\operatorname{miRNA}$ ID $^{1}$ & $P$ & $\begin{array}{c}\text { Fold } \\
\text { Change }\end{array}$ & $\begin{array}{c}\text { PLS } \\
\text { Coefficient }\end{array}$ & ROC-AUC \\
\hline \multicolumn{7}{|c|}{ Upregulated miRNA Transcripts } \\
\hline 1. & hsa-mir-122_hsa-miR-122-5p & hsa-miR-122-5p & $1.06 \times 10^{-9}$ & 2.2135 & $4.71 \times 10^{-2}$ & 0.930 \\
\hline 2. & hsa-mir-3591_hsa-miR-3591-3p & hsa-miR-3591-3p & $1.06 \times 10^{-9}$ & 2.2127 & $4.71 \times 10^{-2}$ & 0.930 \\
\hline 3. & hsa-mir-183_hsa-miR-183-5p & hsa-miR-183-5p & $2.05 \times 10^{-6}$ & 1.9316 & $3.83 \times 10^{-2}$ & 0.855 \\
\hline 4. & hsa-mir-1277_hsa-miR-1277-3p & hsa-miR-1277-3p & $2.13 \times 10^{-5}$ & 1.7727 & $4.04 \times 10^{-2}$ & 0.850 \\
\hline 5. & hsa-mir-548d-1_hsa-miR-548d-3p & hsa-miR-548d-3p & $2.13 \times 10^{-5}$ & 1.6170 & $2.09 \times 10^{-2}$ & 0.859 \\
\hline 6. & hsa-mir-34a_hsa-miR-34a-5p & hsa-miR-34a-5p & $3.81 \times 10^{-5}$ & 1.9308 & $3.45 \times 10^{-2}$ & 0.847 \\
\hline 7. & hsa-mir-576_hsa-miR-576-3p & hsa-miR-576-3p & $3.04 \times 10^{-4}$ & 2.0430 & $3.21 \times 10^{-2}$ & 0.842 \\
\hline 8. & hsa-mir-454_hsa-miR-454-3p & hsa-miR-454-3p & $3.04 \times 10^{-4}$ & 1.2133 & $1.05 \times 10^{-2}$ & 0.833 \\
\hline 9. & hsa-mir-548d-1_hsa-miR-548d-5p & hsa-miR-548d-5p & $3.44 \times 10^{-4}$ & 1.3487 & $1.47 \times 10^{-2}$ & 0.836 \\
\hline 10. & hsa-mir-186_hsa-miR-186-3p & hsa-miR-186-3p & $3.61 \times 10^{-4}$ & 1.3568 & $1.65 \times 10^{-2}$ & 0.814 \\
\hline 11. & hsa-mir-548d-2_hsa-miR-548d-5p & hsa-miR-548d-5p & $3.61 \times 10^{-4}$ & 1.3498 & $1.47 \times 10^{-2}$ & 0.811 \\
\hline 12. & hsa-mir-548aa-1_hsa-miR-548aa & hsa-miR-548aa & $5.13 \times 10^{-4}$ & 1.3248 & $1.46 \times 10^{-2}$ & 0.819 \\
\hline 13. & hsa-mir-548aa-2_hsa-miR-548aa & hsa-miR-548aa & $1.02 \times 10^{-3}$ & 1.3381 & $1.46 \times 10^{-2}$ & 0.797 \\
\hline 14. & hsa-mir-33a_hsa-miR-33a-5p & hsa-miR-33a-5p & $1.02 \times 10^{-3}$ & 1.2067 & $1.13 \times 10^{-2}$ & 0.816 \\
\hline 15. & hsa-mir-590_hsa-miR-590-3p & hsa-miR-590-3p & $1.02 \times 10^{-3}$ & 1.1660 & $6.74 \times 10^{-3}$ & 0.816 \\
\hline 16. & hsa-mir-548t_hsa-miR-548t-3p & hsa-miR-548t-3p & $1.81 \times 10^{-3}$ & 1.3233 & $8.10 \times 10^{-3}$ & 0.796 \\
\hline 17. & hsa-mir-1277_hsa-miR-1277-5p & hsa-miR-1277-5p & $1.84 \times 10^{-3}$ & 1.3291 & $2.13 \times 10^{-2}$ & 0.811 \\
\hline 18. & hsa-let-7b_hsa-let-7b-3p & hsa-let-7b-3p & $2.06 \times 10^{-3}$ & 1.3223 & $1.09 \times 10^{-2}$ & 0.791 \\
\hline 19. & hsa-mir-96_hsa-miR-96-5p & hsa-miR-96-5p & $3.73 \times 10^{-3}$ & 2.2914 & $2.64 \times 10^{-2}$ & 0.786 \\
\hline 20. & hsa-mir-548ac_hsa-miR-548ac & hsa-miR-548ac & $5.53 \times 10^{-3}$ & 1.7613 & $2.87 \times 10^{-2}$ & 0.807 \\
\hline 21. & hsa-mir-19a_hsa-miR-19a-3p & hsa-miR-19a-3p & $5.82 \times 10^{-3}$ & 1.1944 & $8.38 \times 10^{-3}$ & 0.757 \\
\hline 22. & hsa-mir-206_hsa-miR-206 & hsa-miR-206 & $8.00 \times 10^{-3}$ & 2.0356 & $2.76 \times 10^{-2}$ & 0.759 \\
\hline 23. & hsa-mir-497_hsa-miR-497-3p & hsa-miR-497-3p & $9.31 \times 10^{-3}$ & 1.4368 & $1.63 \times 10^{-2}$ & 0.782 \\
\hline 24. & hsa-mir-208a_hsa-miR-208a-3p & hsa-miR-208a-3p & $9.81 \times 10^{-3}$ & 3.2080 & $2.77 \times 10^{-2}$ & 0.789 \\
\hline \multicolumn{7}{|c|}{ Downregulated miRNA transcripts } \\
\hline 25. & hsa-mir-92a-1_hsa-miR-92a-3p & hsa-miR-92a-3p & $7.89 \times 10^{-5}$ & 0.8323 & $-1.40 \times 10^{-2}$ & 0.856 \\
\hline 26. & hsa-mir-874_hsa-miR-874-5p & hsa-miR-874-5p & $1.29 \times 10^{-4}$ & 0.5428 & $-3.43 \times 10^{-2}$ & 0.916 \\
\hline 27. & hsa-mir-106b_hsa-miR-106b-3p & hsa-miR-106b-3p & $2.47 \times 10^{-4}$ & 0.7964 & $-1.15 \times 10^{-2}$ & 0.902 \\
\hline 28. & hsa-mir-92a-2_hsa-miR-92a-3p & hsa-miR-92a-3p & $3.04 \times 10^{-4}$ & 0.8414 & $-1.43 \times 10^{-2}$ & 0.842 \\
\hline 29. & hsa-mir-181a-2_hsa-miR-181a-2-3p & hsa-miR-181a-2-3p & $1.02 \times 10^{-3}$ & 0.6772 & $-3.24 \times 10^{-2}$ & 0.793 \\
\hline 30. & hsa-mir-128-1_hsa-miR-128-3p & hsa-miR-128-3p & $2.67 \times 10^{-3}$ & 0.8504 & $-7.84 \times 10^{-3}$ & 0.777 \\
\hline 31. & hsa-mir-769_hsa-miR-769-5p & hsa-miR-769-5p & $5.53 \times 10^{-3}$ & 0.8706 & $-1.15 \times 10^{-2}$ & 0.794 \\
\hline 32. & hsa-mir-30e_hsa-miR-30e-3p & hsa-miR-30e-3p & $5.53 \times 10^{-3}$ & 0.7400 & $-1.51 \times 10^{-2}$ & 0.805 \\
\hline 33. & hsa-mir-1250_hsa-miR-1250-5p & hsa-miR-1250-5p & $8.56 \times 10^{-3}$ & 0.6186 & $-3.32 \times 10^{-2}$ & 0.803 \\
\hline 34. & hsa-mir-25_hsa-miR-25-3p & hsa-miR-25-3p & $8.94 \times 10^{-3}$ & 0.8603 & $-9.00 \times 10^{-3}$ & 0.766 \\
\hline
\end{tabular}

${ }^{1}$ According to miRBase 22 (http://www.mirbase.org/). These 34 miRNA transcripts result in 31 miRNAs (miRNA IDs). $P$ (FDR with Benjamini-Hochberg correction) and fold change values were obtained from DESeq2 analysis. Partial Least Squares (PLS) coefficients were obtained from Uninformative Variable Elimination by Partial Least Squares (UVE-PLS) analysis. Areas under Receiver Operating Characteristics (ROC) curves (ROC-AUC) were received from ROC analysis. MiRNA transcripts were ordered according to increasing $p$ values across groups of upregulated and downregulated miRNA transcripts. 
ROC analysis revealed that areas under ROC curves for 34 selected miRNA transcripts were covered in the range $0.930-0.757$, indicating a high ability to distinguish patients with CVD from healthy subjects (Table 2, Table S5 and Figure S5).

Correlation analysis between age and expression data of 34 selected miRNA transcripts was performed in CVD group in order to evaluate effect of age on these miRNAs (Table S6). Only hsa-miR-548ac was statistically significantly correlated with age $(p=0.0395)$ exhibiting weak and negative correlation $(R=-0.35)$. The lack of statistically significant correlation of remaining 33 miRNA transcripts suggests their independency from age; however, further studies with larger populations should be performed to confirm this result.

\subsection{Differential Expression Analysis of Genes}

From CVD patient and non-CVD control groups, seven patients and seven controls were randomly selected for gene expression analysis. Similarly to miRNA, DESeq2 and UVE-PLS methods were applied to perform differential gene expression analysis. Significantly dysregulated genes revealed by both methods were selected.

DESeq2 analysis disclosed 23,204 differentially expressed genes in CVD subjects, comparing to controls. In total, 2719 genes presented statistical significance $(p<0.05)$. The risk of false positive results was decreased by selection of 183 differentially expressed genes with $p<0.00001$ (Table S7).

Application of UVE-PLS analysis to gene expression data of 23,204 differentially expressed genes in CVD subjects compared to controls disclosed 74 informative genes (Table S8). Plot presenting the arrangement of prediction error and PLS components as well as plot of cross-validated predictions versus measured values were shown on Figure S6.

The set of 183 differentially expressed genes identified by DESeq2 method with $p<0.00001$ and the set of 74 informative genes identified by UVE-PLS method were compared on a Venn diagram, revealing 62 genes common for both sets (Figure 3a). These 62 common genes constitute a proposed panel of potential biomarkers of CVD (Table 3). Clustering patterns of 62 genes in CVD subjects and controls was visualized on 3D PCA plot and in heatmap with Euclidean clustering (Figure 3b,c, respectively).

The ROC analysis showed that areas under ROC curves obtained for 62 selected genes were equal to 0.98 for $R A C 1 P 2$ and $R P 11-318 C 24.1$, and equal to one for the remaining 60 genes, indicating good precision of CVD classification (Table 3, Table S9, Figure S7).

Correlation analysis between age and expression data of 62 selected genes was performed in CVD group in order to evaluate effect of age on these genes (Table S10). HSPA8P1 ( $\mathrm{R}=-0.82, p=0.024)$, PTBP1P $(\mathrm{R}=-0.80, p=0.031)$, TSC2 $(\mathrm{R}=-0.79, p=0.033)$ and UBA52P5 $(\mathrm{R}=-0.77, p=0.043)$ were statistically significantly and negatively correlated with age. Among these four genes, HSPA8P1, PTBP1P and UBA52P5 were disclosed as downregulated in CVD group, pointing to them as possible age-associated risk factors of CVD. A lack of statistically significant correlation of remaining 58 genes suggests their independency from age; however, further studies with larger populations should be performed to confirm this result.

To estimate the influence of cell subpopulations diversity in the PBMCs samples on results of gene expression analysis, the deconvolution procedure was carried out using "quanTIseq" and "MCPcounter" methods implemented in immunedeconv package. Both methods enable to perform comparisons between samples and "quanTIseq" allows also to make comparisons between cell types. Application of both methods to the gene expression data showed estimated proportions of 11 cell subpopulations in studied samples (Figures S8 and S9). Although differences in proportions of particular cell subpopulations could be observed between samples, our data suggests that there is no significant impact of cell subpopulations composition in PBMCs samples on the study results. 
Table 3. The set of 62 differentially expressed genes in seven patients with chronic venous disease vs. seven controls, resulted from DESeq2 analysis $(p<0.00001)$ with statistical significance confirmed by Uninformative Variable Elimination by Partial Least Squares (UVE-PLS) analysis.

\begin{tabular}{|c|c|c|c|c|c|c|}
\hline No. & Gene Symbol & Gene Name & $p$ Value & $\begin{array}{c}\text { Fold } \\
\text { Change }\end{array}$ & $\begin{array}{c}\text { PLS } \\
\text { Coefficient }\end{array}$ & ROC-AUC \\
\hline \multicolumn{7}{|c|}{ Upregulated Genes } \\
\hline 1. & TSC2 & TSC complex subunit 2 & $4.87 \times 10^{-17}$ & 1.437 & $8.197 \times 10^{-4}$ & 1.000 \\
\hline 2. & $T B C 1 D 22 A$ & TBC1 domain family member 22A & $4.36 \times 10^{-11}$ & 1.431 & $7.572 \times 10^{-4}$ & 1.000 \\
\hline 3. & PPP6R2 & $\begin{array}{c}\text { protein phosphatase } 6 \text { regulatory } \\
\text { subunit } 2\end{array}$ & $9.52 \times 10^{-9}$ & 1.361 & $6.225 \times 10^{-4}$ & 1.000 \\
\hline 4. & UPF1 & UPF1, RNA helicase and ATPase & $2.82 \times 10^{-7}$ & 1.247 & $5.077 \times 10^{-4}$ & 1.000 \\
\hline 5. & WNK1 & WNK lysine deficient protein kinase 1 & $4.59 \times 10^{-7}$ & 1.258 & $4.134 \times 10^{-4}$ & 1.000 \\
\hline 6. & CDS2 & CDP-diacylglycerol synthase 2 & $5.31 \times 10^{-7}$ & 1.241 & $4.756 \times 10^{-4}$ & 1.000 \\
\hline 7. & $P R R C 2 B$ & proline rich coiled-coil 2B & $1.56 \times 10^{-6}$ & 1.273 & $4.693 \times 10^{-4}$ & 1.000 \\
\hline 8. & HDAC5 & histone deacetylase 5 & $4.89 \times 10^{-6}$ & 1.432 & $5.694 \times 10^{-4}$ & 1.000 \\
\hline 9. & $\begin{array}{l}\text { INTS11 } \\
(\text { CPSF3L) }\end{array}$ & integrator complex subunit 11 & $5.95 \times 10^{-6}$ & 1.246 & $4.683 \times 10^{-4}$ & 1.000 \\
\hline \multicolumn{7}{|c|}{ Downregulated genes } \\
\hline 10. & AC078899.1 & Unmatched & $1.18 \times 10^{-13}$ & 0.393 & $-1.586 \times 10^{-3}$ & 1.000 \\
\hline 11. & RP11-16F15.1 & Unmatched & $1.18 \times 10^{-13}$ & 0.327 & $-2.068 \times 10^{-3}$ & 1.000 \\
\hline 12. & EEF1A1P19 & $\begin{array}{l}\text { eukaryotic translation elongation factor } 1 \\
\text { alpha } 1 \text { pseudogene } 19\end{array}$ & $8.40 \times 10^{-13}$ & 0.500 & $-1.305 \times 10^{-3}$ & 1.000 \\
\hline 13. & PFN1P1 & profilin 1 pseudogene 1 & $4.04 \times 10^{-11}$ & 0.367 & $-1.457 \times 10^{-3}$ & 1.000 \\
\hline 14. & RP4-706A16.3 & Unmatched & $4.36 \times 10^{-11}$ & 0.455 & $-1.394 \times 10^{-3}$ & 1.000 \\
\hline 15. & AC005884.1 & Unmatched & $4.36 \times 10^{-11}$ & 0.401 & $-1.488 \times 10^{-3}$ & 1.000 \\
\hline 16. & CALM2P2 & calmodulin 2 pseudogene 2 & $4.36 \times 10^{-11}$ & 0.386 & $-1.498 \times 10^{-3}$ & 1.000 \\
\hline 17. & HSPA8P1 & $\begin{array}{l}\text { heat shock protein family A (Hsp70) } \\
\text { member } 8 \text { pseudogene } 1\end{array}$ & $4.36 \times 10^{-11}$ & 0.379 & $-1.575 \times 10^{-3}$ & 1.000 \\
\hline 18. & RP11-490H24.5 & Unmatched & $4.36 \times 10^{-11}$ & 0.312 & $-1.508 \times 10^{-3}$ & 1.000 \\
\hline 19. & EIF4A1P10 & $\begin{array}{l}\text { eukaryotic translation initiation factor } \\
\text { 4A1 pseudogene } 10\end{array}$ & $4.66 \times 10^{-11}$ & 0.461 & $-1.286 \times 10^{-3}$ & 1.000 \\
\hline 20. & $R P 11-1033 A 18.1$ & Unmatched & $7.00 \times 10^{-11}$ & 0.381 & $-1.495 \times 10^{-3}$ & 1.000 \\
\hline 21. & EIF3FP3 & $\begin{array}{l}\text { eukaryotic translation initiation factor } 3 \\
\text { subunit F pseudogene } 3\end{array}$ & $1.35 \times 10^{-10}$ & 0.443 & $-1.423 \times 10^{-3}$ & 1.000 \\
\hline 22. & $\begin{array}{l}\text { PDIA3P1 } \\
(P D I A 3 P)\end{array}$ & $\begin{array}{l}\text { protein disulfide isomerase family A } \\
\text { member } 3 \text { pseudogene } 1\end{array}$ & $2.38 \times 10^{-10}$ & 0.465 & $-1.240 \times 10^{-3}$ & 1.000 \\
\hline 23. & HSPA9P1 & $\begin{array}{l}\text { heat shock protein family A (Hsp70) } \\
\text { member } 9 \text { pseudogene } 1\end{array}$ & $2.76 \times 10^{-10}$ & 0.420 & $-1.414 \times 10^{-3}$ & 1.000 \\
\hline 24. & AC007238.1 & Unmatched & $3.62 \times 10^{-10}$ & 0.422 & $-1.398 \times 10^{-3}$ & 1.000 \\
\hline 25. & HNRNPA1P7 & $\begin{array}{c}\text { heterogeneous nuclear } \\
\text { ribonucleoprotein A1 pseudogene } 7\end{array}$ & $3.72 \times 10^{-10}$ & 0.462 & $-1.232 \times 10^{-3}$ & 1.000 \\
\hline 26. & RP11-159C21.4 & Unmatched & $4.81 \times 10^{-10}$ & 0.390 & $-1.552 \times 10^{-3}$ & 1.000 \\
\hline 27. & PABPC3 & poly(A) binding protein cytoplasmic 3 & $1.70 \times 10^{-9}$ & 0.414 & $-1.468 \times 10^{-3}$ & 1.000 \\
\hline 28. & $R P 11-74 E 24.2$ & Unmatched & $1.94 \times 10^{-9}$ & 0.537 & $-1.067 \times 10^{-3}$ & 1.000 \\
\hline 29. & EEF1A1P6 & $\begin{array}{l}\text { eukaryotic translation elongation factor } 1 \\
\text { alpha } 1 \text { pseudogene } 6\end{array}$ & $1.94 \times 10^{-9}$ & 0.441 & $-1.375 \times 10^{-3}$ & 1.000 \\
\hline 30. & XRCC6P2 & $\begin{array}{l}\text { X-ray repair cross complementing } 6 \\
\text { pseudogene } 2\end{array}$ & $2.89 \times 10^{-9}$ & 0.373 & $-1.535 \times 10^{-3}$ & 1.000 \\
\hline 31. & HNRNPKP2 & $\begin{array}{l}\text { heterogeneous nuclear } \\
\text { ribonucleoprotein K pseudogene } 2\end{array}$ & $3.13 \times 10^{-9}$ & 0.424 & $-1.163 \times 10^{\wedge}-3$ & 1.000 \\
\hline 32. & EEF1A1P11 & $\begin{array}{l}\text { eukaryotic translation elongation factor } 1 \\
\text { alpha } 1 \text { pseudogene } 11\end{array}$ & $8.40 \times 10^{-9}$ & 0.448 & $-1.369 \times 10^{-3}$ & 1.000 \\
\hline 33. & UBA52P5 & $\begin{array}{l}\text { ubiquitin A-52 residue ribosomal protein } \\
\text { fusion product } 1 \text { pseudogene } 5\end{array}$ & $8.40 \times 10^{-9}$ & 0.397 & $-1.306 \times 10^{-3}$ & 1.000 \\
\hline 34. & RPL9P7 & ribosomal protein L9 pseudogene 7 & $9.10 \times 10^{-9}$ & 0.414 & $-1.417 \times 10^{-3}$ & 1.000 \\
\hline 35. & RPS21P4 & ribosomal protein S21 pseudogene 4 & $1.37 \times 10^{-8}$ & 0.376 & $-1.531 \times 10^{-3}$ & 1.000 \\
\hline
\end{tabular}


Table 3. Cont.

\begin{tabular}{|c|c|c|c|c|c|c|}
\hline No. & Gene Symbol & Gene Name & $p$ Value & $\begin{array}{l}\text { Fold } \\
\text { Change }\end{array}$ & $\begin{array}{c}\text { PLS } \\
\text { Coefficient }\end{array}$ & ROC-AUC \\
\hline 36. & RP11-334L9.1 & Unmatched & $1.37 \times 10^{-8}$ & 0.333 & $-1.206 \times 10^{-3}$ & 1.000 \\
\hline 37. & HNRNPKP4 & $\begin{array}{l}\text { heterogeneous nuclear } \\
\text { ribonucleoprotein K pseudogene } 4\end{array}$ & $1.38 \times 10^{-8}$ & 0.462 & $-1.120 \times 10^{-3}$ & 1.000 \\
\hline 38. & RPL9P9 & ribosomal protein L9 pseudogene 9 & $1.38 \times 10^{-8}$ & 0.418 & $-1.302 \times 10^{-3}$ & 1.000 \\
\hline 39. & AC138123.2 & Unmatched & $1.38 \times 10^{-8}$ & 0.407 & $-1.422 \times 10^{-3}$ & 1.000 \\
\hline 40. & HNRNPA1P10 & $\begin{array}{l}\text { heterogeneous nuclear } \\
\text { ribonucleoprotein A1 pseudogene } 10\end{array}$ & $1.39 \times 10^{-8}$ & 0.475 & $-1.227 \times 10^{-3}$ & 1.000 \\
\hline 41. & MORF4L1P1 & mortality factor 4 like 1 pseudogene 1 & $3.98 \times 10^{-8}$ & 0.535 & $-1.045 \times 10^{-3}$ & 1.000 \\
\hline 42. & $R P 11-676 M 6.1$ & Unmatched & $8.19 \times 10^{-8}$ & 0.498 & $-1.208 \times 10^{-3}$ & 1.000 \\
\hline 43, & RPL7AP66 & ribosomal protein L7a pseudogene 66 & $9.71 \times 10^{-8}$ & 0.485 & $-1.089 \times 10^{-3}$ & 1.000 \\
\hline 44. & RP11-680H20.1 & Unmatched & $9.99 \times 10^{-8}$ & 0.411 & $-1.155 \times 10^{-3}$ & 1.000 \\
\hline 45. & CTB-13H5.1 & Unmatched & $1.41 \times 10^{-7}$ & 0.418 & $-1.175 \times 10^{-3}$ & 1.000 \\
\hline 46. & HNRNPA1P35 & $\begin{array}{l}\text { heterogeneous nuclear } \\
\text { ribonucleoprotein A1 pseudogene } 35\end{array}$ & $1.49 \times 10^{-7}$ & 0.350 & $-1.223 \times 10^{-3}$ & 1.000 \\
\hline 47. & PTBP1P & $\begin{array}{l}\text { polypyrimidine tract binding protein } 1 \\
\text { pseudogene }\end{array}$ & $1.53 \times 10^{-7}$ & 0.443 & $-1.095 \times 10^{-3}$ & 1.000 \\
\hline 48. & API5P1 & apoptosis inhibitor 5 pseudogene 1 & $1.57 \times 10^{-7}$ & 0.347 & $-1.204 \times 10^{-3}$ & 1.000 \\
\hline 49. & UBE2D3P1 & $\begin{array}{l}\text { ubiquitin conjugating enzyme E2 D3 } \\
\text { pseudogene } 1\end{array}$ & $1.69 \times 10^{-7}$ & 0.485 & $-8.801 \times 10^{-4}$ & 1.000 \\
\hline 50. & AL162151.3 & Unmatched & $1.94 \times 10^{-7}$ & 0.431 & $-1.258 \times 10^{-3}$ & 1.000 \\
\hline 51. & RPL9P8 & ribosomal protein L9 pseudogene 8 & $2.34 \times 10^{-7}$ & 0.446 & $-1.253 \times 10^{-3}$ & 1.000 \\
\hline 52. & EEF1A1P13 & $\begin{array}{l}\text { eukaryotic translation elongation factor } 1 \\
\text { alpha } 1 \text { pseudogene } 13\end{array}$ & $2.51 \times 10^{-7}$ & 0.521 & $-1.211 \times 10^{-3}$ & 1.000 \\
\hline 53. & PABPC1P4 & $\begin{array}{l}\text { poly(A) binding protein cytoplasmic } 1 \\
\text { pseudogene } 4\end{array}$ & $2.60 \times 10^{-7}$ & 0.465 & $-1.031 \times 10^{-3}$ & 1.000 \\
\hline 54. & HNRNPUP1 & $\begin{array}{l}\text { heterogeneous nuclear } \\
\text { ribonucleoprotein U pseudogene } 1\end{array}$ & $2.73 \times 10^{-7}$ & 0.441 & $-1.106 \times 10^{-3}$ & 1.000 \\
\hline 55. & ARPC3P1 & $\begin{array}{l}\text { actin related protein } 2 / 3 \text { complex subunit } \\
\qquad 3 \text { pseudogene } 1\end{array}$ & $3.72 \times 10^{-7}$ & 0.331 & $-1.272 \times 10^{-3}$ & 1.000 \\
\hline 56. & PTP4A2P1 & $\begin{array}{l}\text { protein tyrosine phosphatase type IVA, } \\
\text { member } 2 \text { pseudogene } 1\end{array}$ & $4.59 \times 10^{-7}$ & 0.500 & $-9.014 \times 10^{-4}$ & 1.000 \\
\hline 57. & CTC-451P13.1 & Unmatched & $4.77 \times 10^{-7}$ & 0.513 & $-9.263 \times 10^{-4}$ & 1.000 \\
\hline 58. & $B Z W 1 P 2$ & $\begin{array}{l}\text { basic leucine zipper and W2 domains } 1 \\
\text { pseudogene } 2\end{array}$ & $7.97 \times 10^{-7}$ & 0.445 & $-9.598 \times 10^{-4}$ & 1.000 \\
\hline 59. & $R P 11-318 C 24.1$ & Unmatched & $1.94 \times 10^{-6}$ & 0.314 & $-1.062 \times 10^{-3}$ & 0.980 \\
\hline 60. & $\begin{array}{l}\text { OTUD4P1 } \\
(\text { HIN1L) }\end{array}$ & OTUD4 pseudogene 1 & $2.08 \times 10^{-6}$ & 0.480 & $-9.962 \times 10^{-4}$ & 1.000 \\
\hline 61. & EIF3LP2 & $\begin{array}{l}\text { eukaryotic translation initiation factor } 3 \\
\text { subunit L pseudogene } 2\end{array}$ & $2.33 \times 10^{-6}$ & 0.457 & $-9.995 \times 10^{-4}$ & 1.000 \\
\hline 62. & $R A C 1 P 2$ & Rac family small GTPase 1 pseudogene 2 & $3.29 \times 10^{-6}$ & 0.489 & $-8.192 \times 10^{-4}$ & 0.980 \\
\hline
\end{tabular}

$P$ (FDR with Benjamini-Hochberg correction) and fold change values were obtained from DESeq 2 analysis. PLS coefficients were obtained from UVE-PLS analysis. Areas under Receiver Operating Characteristics (ROC) curves (ROC-AUC) were received from ROC analysis. Genes were ordered according to increasing $p$ values across groups of upregulated and downregulated genes. Genes without names assigned by HUGO Multi-symbol checker were termed as "Unmatched". Synonyms or previous gene symbols were put into brackets. 
a

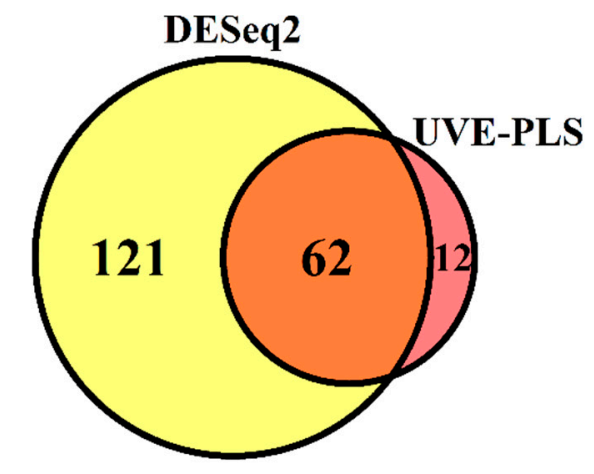

b

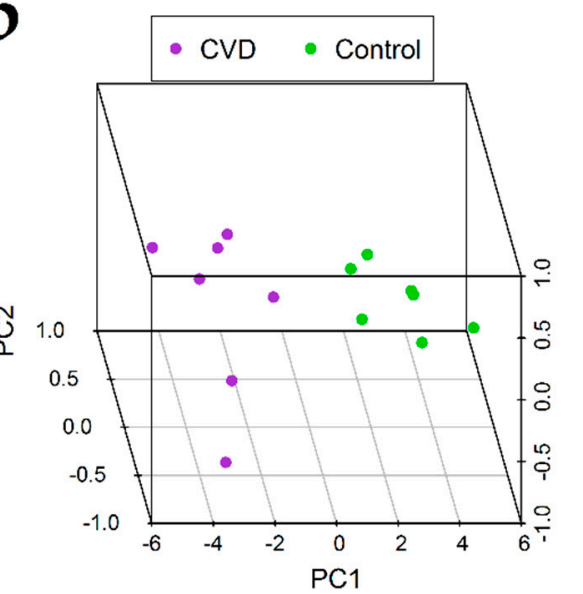

C

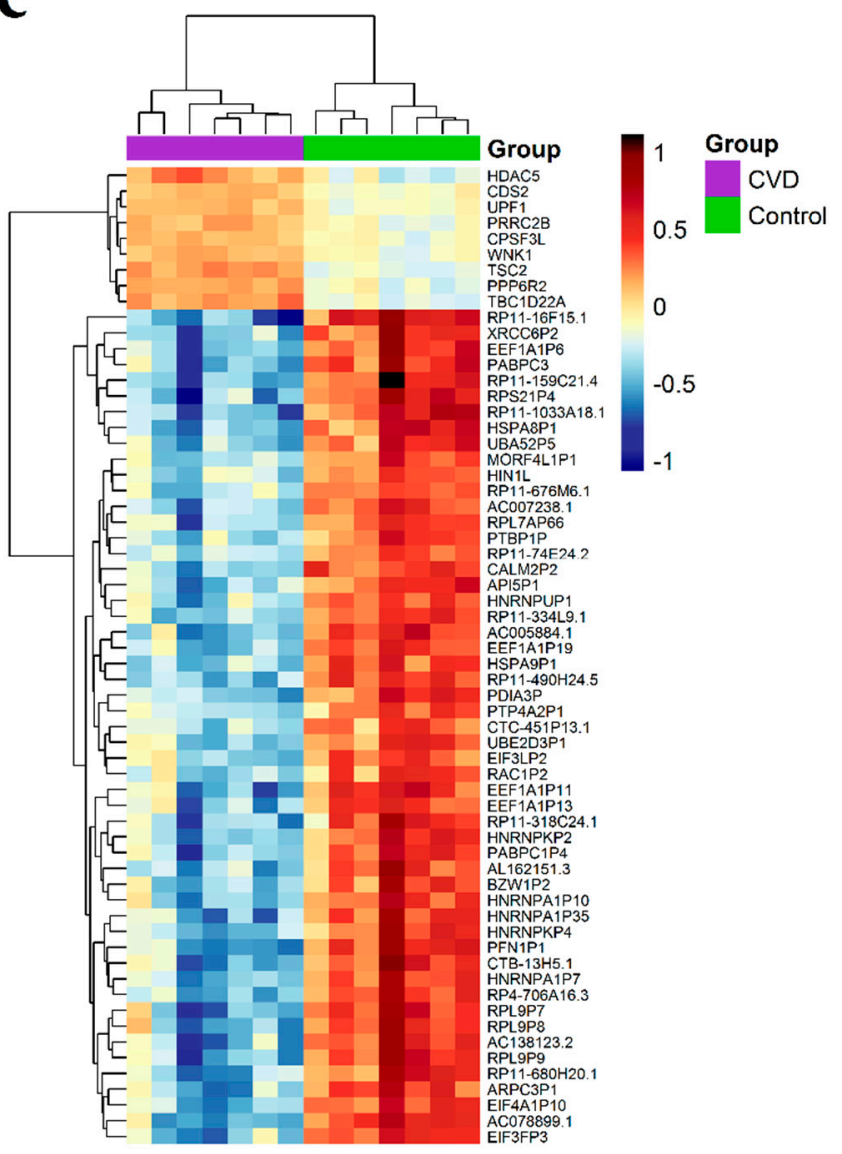

Figure 3. Results of differential expression analysis of genes in group of seven patients with chronic venous disease (CVD) vs. seven healthy controls group (Control). (a) Venn diagram presenting comparison of two gene sets: the set of 183 genes received from DESeq2 analysis with $p<0.00001$ and the set of 74 informative genes indicated by Uninformative Variable Elimination by Partial Least Squares (UVE-PLS) analysis. Sixty-two genes were common for both sets of genes. 3D Principal Component Analysis (PCA) plot (b) and heatmap with Euclidean clustering (c) show differential expression of common 62 genes in CVD and Control groups.

\subsection{In Silico Identification of miRNA:Gene Interactions}

Identification of miRNA:gene interactions between 31 selected miRNAs and 62 selected genes was performed in silico by multiMiR package. Twelve validated (Table S11) and 51 top 10\%-predicted miRNA:gene pairs (Table S12) were returned from analysis. Interactions between miRNAs and their targets were visualized as a regulatory network containing 22 miRNAs and seven genes (Figure 4). 


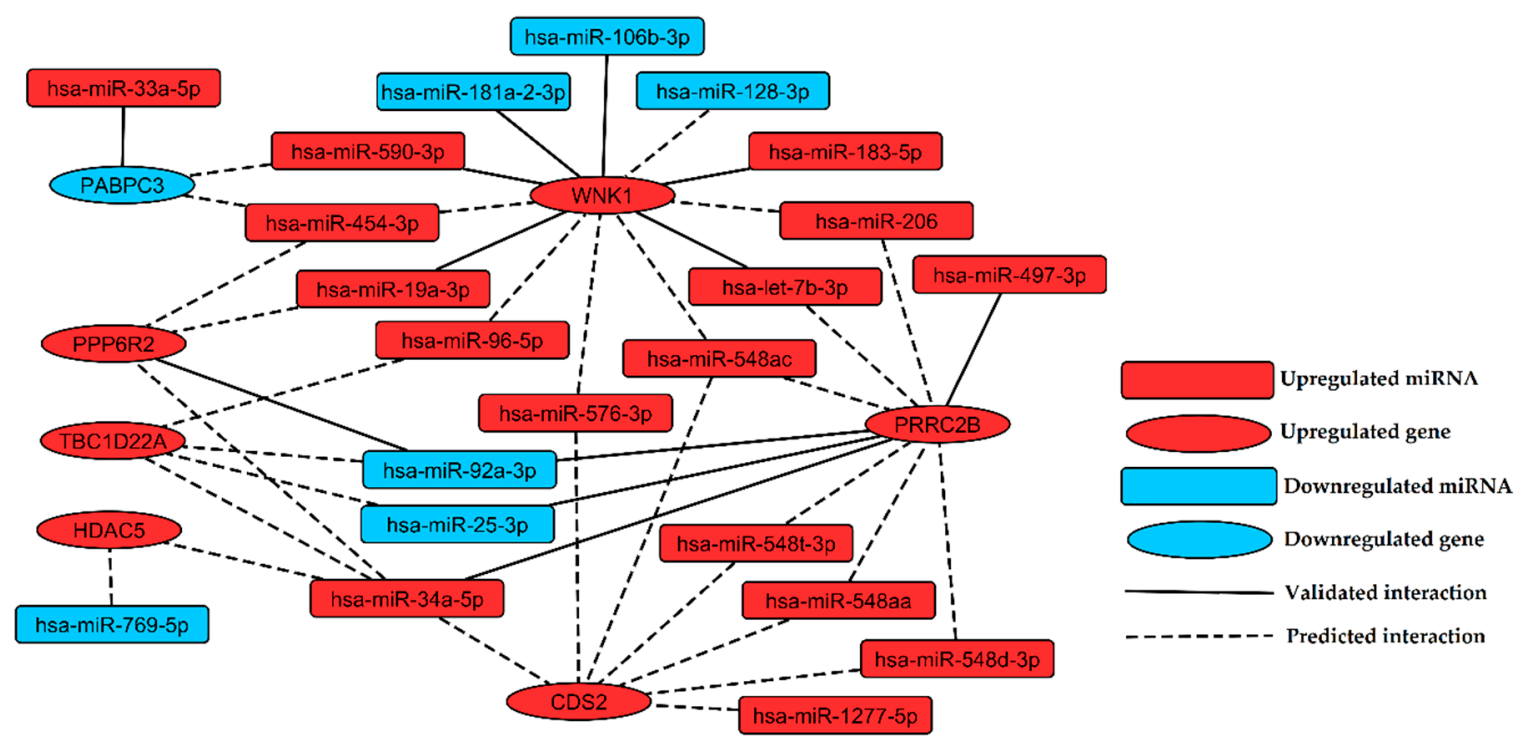

Figure 4. Regulatory network of interactions found in silico between miRNAs and genes indicated as the most promising biomarkers of chronic venous disease. Upregulated and downregulated nodes (miRNAs or genes) were labeled with red and blue color, respectively. Validated and predictive interactions were labeled with solid and dashed edges, respectively.

\subsection{Functional Analysis of miRNA Targets}

Functional analysis was performed using DAVID 6.8 tool for seven networked target genes (CDS2, HDAC5, PPP6R2, PRRC2B, TBC1D22A, WNK1, and PABPC3). Analyzed genes were associated with cardiovascular diseases and risk factors (TBC1D22A, WNK1), bone mineral density (HDAC5), body weight (PABPC3), glycerophospholipid metabolism (CDS2), Notch signaling (HDAC5), RNA transport and degradation (PABPC3). Three genes: CDS2, TBC1D22A, and WNK1 were connected to tobacco use disorder, which may be caused by the prevalence of smoking in $14.7 \%$ of CVD population (control group consists of non-smoking individuals, Table 1). GO enrichment analysis assigned upregulated genes (CDS2, HDAC5, PPP6R2, PRRC2B, TBC1D22A, and WNK1) to developmental processes and downregulated gene PABPC3 to RNA metabolic processes (Table 4).

Table 4. Results of functional analysis of seven genes selected in silico as targets of miRNA identified as signatures of chronic venous disease.

\begin{tabular}{ll}
\hline \multicolumn{2}{c}{ Functional Analysis of Upregulated Genes (CDS2, HDAC5, PPP6R2, PRRC2B, TBC1D22A, WNK1) } \\
\hline \multicolumn{1}{c}{ KEGG, Reactome, GAD and GAD Class } \\
\hline KEGG: Glycerophospholipid metabolism, Phosphatidylinositol signaling system, \\
Metabolic pathways \\
Reactome: Synthesis of PG (Phosphatidylglycerol) \\
GAD: Type 2 Diabetes|edema|rosiglitazone, Tobacco Use Disorder \\
GAD Class: pharmacogenomic, chemdependency \\
KEGG: Alcoholism, Viral carcinogenesis, \\
Reactome: NOTCH1 Intracellular Domain Regulates Transcription, Constitutive Signaling \\
by NOTCH1 PEST Domain Mutants, Constitutive Signaling by NOTCH1 HD + PEST \\
Domain Mutants \\
GAD: antidepressant response, Bone Density, Bone mineral density (hip), Bone mineral \\
density (spine), bronchodilator response, Fractures, Bone, Type 2 Diabetes| edema | \\
rosiglitazone \\
GAD Class: immune, metabolic, pharmacogenomic
\end{tabular}


Table 4. Cont.

\begin{tabular}{|c|c|}
\hline \multicolumn{2}{|c|}{ Functional Analysis of Upregulated Genes (CDS2, HDAC5, PPP6R2, PRRC2B, TBC1D22A, WNK1) } \\
\hline PPP6R2 & No information \\
\hline PRRC2B & No information \\
\hline TBC1D22A & $\begin{array}{l}\text { GAD: Albumins, Arteries, Attention Deficit Disorder with Hyperactivity, Blood Pressure, } \\
\text { Body Mass Index, Body Weight, Breath Tests, Cardiomegaly, Cholesterol, Erythrocyte } \\
\text { Count, Fibrinogen, Heart Failure, Heart Rate, Leukocyte Count, longevity, Metabolism, } \\
\text { Myocardial Infarction, Parkinson Disease, Resistin, Stroke, Thyrotropin, Tobacco Use } \\
\text { Disorder, Waist Circumference, Waist-Hip Ratio } \\
\text { GAD Class: aging, cardiovascular, chemdependency, hematological, immune, metabolic, } \\
\text { neurological, other, psych }\end{array}$ \\
\hline WNK1 & $\begin{array}{l}\text { Reactome: Stimuli-sensing channels } \\
\text { GAD: Apoplexy|Brain Ischemia|Stroke, blood pressure, arterial, Chronic renal } \\
\text { failure|Kidney Failure, Chronic, Essential Hypertension, Hereditary Sensory and } \\
\text { Autonomic Neuropathies, HIV Infections|[X]Human immunodeficiency virus disease, } \\
\text { hypertension, null, Tobacco Use Disorder, Type } 2 \text { Diabetes| edema | rosiglitazone } \\
\text { GAD Class: cardiovascular, chemdependency, infection, neurological, pharmacogenomic, } \\
\text { renal, unknown }\end{array}$ \\
\hline \multicolumn{2}{|r|}{ Gene Ontology terms associated with EASE score $<0.1$} \\
\hline GO Biological Process & cellular developmental process, positive regulation of molecular function \\
\hline GO Molecular Function & enzyme binding \\
\hline \multicolumn{2}{|r|}{ Functional analysis of downregulated gene (PABPC3) } \\
\hline \multicolumn{2}{|r|}{ KEGG, Reactome, GAD and GAD Class } \\
\hline РАВРC3 & $\begin{array}{l}\text { KEGG: RNA transport, mRNA surveillance pathway, RNA degradation } \\
\text { GAD: Body Mass Index, Body Weight, Body Weight Changes, Glomerular Filtration Rate } \\
\text { GAD Class: metabolic, renal }\end{array}$ \\
\hline \multicolumn{2}{|r|}{ Gene Ontology terms associated with PABPC3 } \\
\hline GO Biological Process & $\begin{array}{l}\text { nucleobase-containing compound metabolic process, cellular aromatic compound } \\
\text { metabolic process, nitrogen compound metabolic process, metabolic process, cellular } \\
\text { process, RNA metabolic process, mRNA metabolic process, cellular nitrogen compound } \\
\text { metabolic process, macromolecule metabolic process, cellular metabolic process, primary } \\
\text { metabolic process, cellular macromolecule metabolic process, heterocycle metabolic } \\
\text { process, organic substance metabolic process, nucleic acid metabolic process, organic cyclic } \\
\text { compound metabolic process }\end{array}$ \\
\hline GO Molecular Function & $\begin{array}{l}\text { nucleotide binding, nucleic acid binding, RNA binding, single-stranded RNA binding, } \\
\text { binding, poly(A) binding, small molecule binding, poly-purine tract binding, organic cyclic } \\
\text { compound binding, nucleoside phosphate binding, heterocyclic compound binding }\end{array}$ \\
\hline $\begin{array}{l}\text { GO Cellular } \\
\text { Component }\end{array}$ & $\begin{array}{l}\text { extracellular region, intracellular, cell, cytoplasm, vesicle, membrane-bounded vesicle, } \\
\text { organelle, membrane-bounded organelle, extracellular organelle, extracellular region part, } \\
\text { intracellular part, cell part, extracellular exosome, extracellular vesicle }\end{array}$ \\
\hline $\begin{array}{l}\text { Analysis was carried } \\
\text { Genes and Genomes }\end{array}$ & $\begin{array}{l}\text { with DAVID } 6.8 \text { website tool and all associated functional terms of Kyoto Encyclopedia of } \\
\text { GG), Reactome, Genetic Association Database (GAD), Genetic Association Database Class } \\
\text { are presented. Gene Ontology (GO) terms associated with upregulated genes with EASE } \\
\text { ted. For downregulated gene, all associated GO terms were presented. }\end{array}$ \\
\hline
\end{tabular}

\section{Discussion}

High prevalence, multifactorial character and diverse symptomatology of CVD make this disease one of the major health problems worldwide [49]. There is a lack of sensitive and specific biomarkers for early detection of CVD and for monitoring disease progression. Altered expression of miRNA and its effects on regulation of gene expression make it a promising candidate for novel diagnostic and treatment approaches.

In the presented study, we conducted integrated, comparative analysis of miRNA and gene expression in PBMCs of patients with CVD and healthy controls. We applied Next Generation Sequencing and various statistical and bioinformatic tools to analyze expression profiles of CVD patients vs. controls and to search for genetic signatures of CVD (Figure 1). Most promisingly, 31 miRNAs (Table 2) and 62 genes (Table 3), which potentially may serve as biomarkers for CVD, 
were selected. The discriminative character of proposed biomarkers was reinforced by decreasing the $p$ value of statistical significance in DESeq2 analysis $(p<0.01$ for miRNAs and $p<0.00001$ for genes, refer to Tables 2 and 3, respectively) and elimination of uninformative variables using the UVE-PLS method. In ROC analysis, we confirmed very solid diagnostic value of proposed biomarkers (Tables 2 and 3, Tables S5 and S9, Figures S5 and S7). This multi-step procedure with strict criteria was applied to obtain scientifically valid results and to eliminate RT-qPCR validation.

The presented miRNA and gene signatures of CVD expand the diagnostic perspective; however, the study population is relatively minor, especially in case of transcriptomic analysis and evaluation with larger cohorts is required to confirm diagnostic utility and discriminative ability of proposed CVD biomarkers.

Integration of miRNA and gene expression analysis in this study allowed us to determine the framework of miRNA regulatory network in CVD, introducing experimentally validated and predictive interactions between significantly differentially expressed miRNA and genes found in patients with CVD (Figure 4). The findings of this study expand our understanding of miRNA functions and provide more insights into a complex network of post-transcriptional control in CVD.

To date, the topic of miRNA and gene expression analysis in venous disorders has not been sufficiently studied [33,34]. Cui et al. used microarray and RT-PCR to research upregulation of miR-34a, miR-202 and downregulation of miR-155 in vein biopsies from patients with CVI [33]. Our study confirmed that upregulated expression of miR-34a is indicative for chronic venous disorder.

Significantly higher expression of miR-16, miR-20a, miR-21, miR-106a, miR-203 and miR-130a was reported in skin biopsies from venous ulcers, comparing to normal skin specimens. Overexpression of these miRNAs was associated with inhibition of wound healing through targeting mRNA for EGR3, $\mathrm{Vcl}$ and LepR. Administration of miR-21 mimics in rat acute wound healing models leads to increase in infiltration of immune cells and reduction of epithelialization [34]. In our study, we demonstrated statistically significant upregulation of miR-21 (Table S3) in CVD subjects with varicose veins, which can be classified as an early stage of the disease. Therefore, enhanced signaling of miR-21 appears to be a factor involved in CVD progression both in early and advanced stages of disease development.

In other studies, miR-34a and miR-21 were significantly upregulated in plasma and in atherosclerotic plaques of patients with CAD (Coronary Artery Disease) [50,51], suggesting that overexpression of miR-34a and miR-21 also found in our study to is common for both arterial and venous pathology. Diagnostic and prognostic potential of increased level of miR-21 was also reported as oncomiR in many types of cancer, including Hodgkin lymphoma [52], head and neck squamous cell carcinoma [53] and lung cancer [54,55], while miR-34a acts as tumor suppressor and undergo epigenetic silencing in carcinogenesis [56].

Some studies indicated altered expression of genes in veins obtained from patients with CVD and venous ulcers [57-60] but none of the genes indicated in abovementioned research were found in our study. These differences could be related to the biological material type, inclusion criteria and methodological approach.

Presented miRNA regulatory network shows that upregulation of WNK1 is associated with downregulation of miR-181a-2-3p and miR-106b-3p (Figure 4). WNK1 is important for proper proliferation, chemotaxis and invasion of endothelial cells [61]. Deficiency of the WNK1 gene in mice induces embryonic lethality due to angiogenic and cardiovascular defects [61]. MiRNAs belonging to miR-181 family play a key role in vascular inflammation through regulation of NF- $\kappa B$ signaling, activation of endothelial cells and homeostasis of immune cells [62]. Upregulation of miR-181a in THP-1 cells exposed to oxidated LDL, lipopolysaccharides and phorbol myristate leads to reduction of foam cells formation and inflammatory cytokines levels, lower production of reactive oxygen species and inhibition of apoptosis $[63,64]$. A higher level of miR-181a in plasma was proposed as a biomarker of myocardial infarction [65]. Overexpression of miR-106b-3p was previously described in the late stage of endothelial replicative senescence [66]. Downregulation of miR-106b-3p was observed in human retinal pigment epithelium cells (ARPE-19) exposed to hydrogen peroxide $\left(\mathrm{H}_{2} \mathrm{O}_{2}\right)$ [67]. Therefore, 
we hypothesize that upregulation of WNK1 observed in our study, associated with downregulation of miR-181a-2-3p and miR-106b-3p is a possible mechanism promoting inflammation and aging in response to oxidative stress in CVD.

WNK1 was identified in our study as a putative target for downregulated miR-128-3p (Figure 4). Zhou et al. showed that downregulation of miR-128-3p promotes endothelial cell proliferation through $\mathrm{Ca}^{2+}$ and ERK1/2-Akt signaling [68]. Upregulation of WNK1 as a consequence of miR-128-3p downregulation may constitute the mechanism promoting endothelial cell proliferation in CVD. Downregulation of miR-128-3p could also be a factor alleviating inflammation, since it was reported to stimulate inflammation via targeting the TNFAIP3 gene and enhancing NF- $\mathrm{KB}$ signaling [69].

MiRNA regulatory network of CVD (Figure 4) shows that miR-33a-5p targets PABPC3, which is a gene encoding a putative regulator of MEG3 stability [70]. MEG3 is a suppressor of tumor growth, inhibiting proliferation and promoting apoptosis in cancer cells [71] via activation of p53 [72]. Our study points to downregulation of $P A B P C 3$ with accompanying overexpression of miR-33a-5p as a factor which may affect MEG3 stability and in consequence promote cell proliferation in CVD.

Another upregulated miRNA found in CVD subjects, associated with MEG3 is miR-183-5p. This miRNA was reported to alleviate symptoms of hypoxia (such as a decrease in cell viability and migration) in H9c2 rat cardiomyocytes via targeting genes encoding MEG3 and p27 [73]. Thus, the upregulation of miR-183-5p observed in our study may be considered as another factor enhancing cell proliferation and hypertrophy in CVD. Additionally, in the presented miRNA regulatory network, upregulated miR-183-5p interacts with the upregulated WNK1 gene. This relationship may also lead to a hyper-proliferative effect, since WNK1 has been reported to be required for proliferation of endothelial cells and vascular smooth muscle cells [61,74].

In our study, downregulation of miR-769-5p in CVD patients was observed. Lower expression of this miRNA was reported in cancer cells and was associated with enhanced proliferation and reduced apoptosis $[75,76]$. Downregulation of miR-769-5p was also found in arterial samples of abdominal and popliteal arterial aneurysm [77]. In the miRNA regulatory network presented here, HDAC5 is proposed as a putative target of miR-769-5p. Xu et al. reported that HDAC5 mediates angiotensin II-induced MEF2 activation and vascular smooth muscle cells hypertrophy [78]. These findings suggest that HDAC5 upregulation, most likely resulted from downregulation of miR-769-5p, may be responsible for pathological vascular hypertrophy in CVD.

We indicated upregulation of miR-206 in CVD patients as an important target, which was already revealed to impair viability and migration of endothelial progenitor cells and to promote apoptosis through targeting VEGF [79]. MiR-206 mediate silencing of VEGF leading to inhibition of angiogenesis during Danio rerio development [80]. Upregulation of miR-206 in our study may thus point to inhibition of angiogenesis; however, two genes encoding downstream effectors of VEGF signaling, WNK1 [81] and CDS2 [82], were upregulated in CVD subjects. Upregulation of WNK1 and CDS2 may be a consequence of increase in VEGF expression mediated by chromatin remodeling caused by upregulation of HDAC5 [83,84], which was also observed in the current study. These findings may suggest an increase in angiogenesis in CVD independently from the VEGFR signaling. On the other hand, a different study indicated HDAC5 as a negative regulator of angiogenesis acting through genes for pro-angiogenic factors FGF2 and Slit2 [85], showing that reciprocal interaction network in CVD is more complex and requires further studies to answer all questions.

Downregulation of miR-30e-3p was previously observed in coronary microembolization and was associated with myocardial injury mediated by impairment of autophagy in cardiomyocytes [86]. Therefore, the downregulation of miR-30e-3p found in this study may be involved in vascular dysfunction related to the thrombosis in CVD.

Kim and collaborators reported at least 5-fold higher level of miR-33a in plasma from individuals with high risk of atherosclerosis, compared to non-high-risk subjects [87]. A significantly higher level of this miRNA was also described in PBMCs isolates from patients with CAD and was positively correlated with higher lipid levels and risk of atherosclerosis $[88,89]$. Therefore, the upregulation 
of miR-33a-5p found in our study may indicate higher risk of atherosclerosis in subjects with CVD, compared to non-CVD subjects.

Upregulation of circulating miR-92a-3p was observed in the plasma of both mild cognitive impairment and Alzheimer disease subjects [90] and was proposed as a biomarker of schizophrenia [91]. In our study, downregulated miR-92a-3p putatively targets upregulated PRRC2B (Figure 4), which is a gene encoding protein involved in brain development [92]. It suggests that some elements of neurodevelopmental process may also participate in CVD pathology, showing connections between vascular and neurodegenerative disorders.

The preliminary functional analysis of proposed transcriptomic biomarkers provides useful information on the pathogenesis of CVD. Functional analysis showed the association of three genes (CDS2, TBC1D22A and WNK1) with tobacco use disorder. The discriminative character of these genes in our study may be, to a certain extent, a result of smoking prevalence in the CVD group; however, smoking is also an established risk factor of CVD [2] and altered expression of CDS2, TBC1D22A and WNK1 could be involved in CVD development through smoking-related mechanisms. Many smoking-induced miRNAs were previously identified and associated with various pathological conditions, including carcinogenesis [93]. Presence of other smoking-related diseases was limited by inclusion patients diagnosed exclusively for CVD, with no other, especially cardiovascular, conditions detected during examination.

PBMCs constitute an important element of inflammation process in vascular diseases [11]; thus, transcriptional profiling of this cell pool should provide reliable information pertinent for vascular pathology. Another advantage of PBMCs is their accessibility through minimally invasive procedures, facilitating utilization in basic and clinical research. Despite of all advantages, co-prevalence of other diseases like chronic obstructive pulmonary disease, hypertension, diabetes mellitus and accompanying complications may bias the evaluation of expression profiles on a systemic scale. To cope with this bias, only subjects without co-existing conditions mentioned in the experimental section were included to the study. Such strict evaluation helped us to find systemic regulatory changes in miRNA and gene expression, which potentially are reflective of local changes in CVD. However, application of these exclusion criteria entailed construction of CVD and control groups with statistically significant differences in age and smoking history, which can introduce some biological bias to our results; therefore, further investigations with more balanced population groups should be performed.

We are aware that our research has several limitations. Studied PBMCs samples may differ in proportions of cell subpopulations (lymphocytes, monocytes), which may introduce a bias in miRNA and gene expression patterns. To assess the impact of this factor, we performed a deconvolution procedure using two methods: "quanTIseq" and "MCPcounter" implemented in an immunedeconv package, enabling comparison between cell types and between samples. The results of deconvolution do not indicate that differences in proportions of particular cell subpopulations across samples which included the CVD and control group had a significant impact on the study outcome (Figures S8 and S9).

Due to technical constraints (server capacity), gene expression analysis was performed on the subset of participants subjected to miRNA expression analysis, which may affect the described transcriptomic effect of miRNA expression alterations in CVD patients. Despite this imbalance, we confirmed some previously validated interactions (Table S11) and determined with high probability other connections in signaling network (Table S12). Future studies should also include in vitro and in vivo validation of predictive interactions of presented miRNA regulatory network. Moreover, in the group selected for gene expression analysis, both miRNA and total RNA were isolated from PBMC specimens obtained from exactly the same subjects and represent the same physiological conditions probed at the same time and circumstances.

Despite applying strict criteria to select the most promising signatures of CVD, the biomarker role of particular miRNAs and gene sets should be confirmed in further studies with larger cohorts and with the application of other validation methods, such as RT-qPCR. Further experiments will involve investigations regarding clinical factors (e.g., stage of disease or medications) and elucidation 
of whether dysregulations of miRNAs and genes indicative for CVD were predictive of or responsive to the disease development.

The results obtained in our current study confirm the significance of miRNA-dependent epigenetic regulation in the pathogenesis of CVD. Although we proposed a novel biomarker panel of CVD, there is still a need for further research on the role of miRNA regulation in the CVD due to small sample size as well as a clear exploratory and hypothesis-generating character of the presented experiments.

The presented discoveries may be used in further fundamental research and may prove to be useful for clinicians and practitioners, providing new paths in diagnosis, differentiation and treatment procedures for CVD patients in future.

\section{Conclusions}

Owing to broad and detailed Next Generation Sequencing analysis one is able to draw some general conclusions about CVD. Analysis of microRNAs and genes dysregulated in CVD unveiled numerous terms related to general physiological processes and traits like inflammation, metabolism, aging as well as more specific ones like lipidomics, cardiovascular diseases and chemodependencies. Future research on much numerous groups of patients and controls would broaden our knowledge about cardiovascular diseases, enabling personalized approach to individual patients.

Supplementary Materials: The following are available online at http://www.mdpi.com/2077-0383/9/5/1251/s1, Figure S1: Distribution of age (A), BMI (B), sex (C) and smoking (D) in CVD and control group. Figure S2: Control plots for miRNA sequencing data. Figure S3: Control plots for transcriptome sequencing data. Figure S4: Plots for UVE-PLS analysis of miRNA expression in CVD group in comparison to control group. Figure S5: Receiver Operating Characteristics (ROC) curves for 34 miRNA transcripts selected as signatures of CVD. Figure S6: Plots for UVE-PLS analysis of genes expression in CVD group compared to control group. Figure S7: Receiver Operating Characteristics (ROC) curves for 62 genes selected as signatures of CVD. Figure S8: Results of gene expression deconvolution procedure for seven CVD patients and seven control subjects performed using "quanTIseq" method implemented to immunedeconv 2.0.0 package. Figure S9: Results of deconvolution procedure performed on gene expression datasets of 7 CVD patients (CVD) and 7 control subjects (Control) using "MCPcounter" method implemented to immunedeconv 2.0.0 package. Table S1: Measurements of small RNA samples and small RNA libraries as well as results of small RNA sequencing data analysis received from Ion Torrent small RNA Plugin v5.0.5r3. Table S2: Measurements of transcriptome libraries and results of transcriptome sequencing data analysis received from Ion Torrent RNASeqAnalysis plugin v.5.0.3.0. Table S3: The set of 96 differentially expressed microRNA transcripts resulted from DESeq2 analysis with $p<0.05$ in 34 patients with CVD compared to 19 controls. Table S4: The set of 48 differentially expressed microRNA transcripts resulted from UVE-PLS analysis in in 34 patients with CVD group compared to 19 controls. Table S5: Results of ROC analysis for 34 miRNA transcripts selected as indicative for CVD. Table S6: Correlation analysis between age and expression of 34 selected miRNA transcripts in CVD group. Table S7: 183 differentially expressed genes resulted from DESeq2 analysis with $p<0.00001$ in seven patients with CVD, compared to seven controls. Table S8: The set of 74 differentially expressed genes resulted from UVE-PLS analysis in seven patients with CVD compared to seven controls. Table S9: Results of ROC analysis for 62 genes selected as indicative for CVD. Table S10: Correlation analysis between age and expression of 62 selected genes in CVD group. Table S11: Twelve experimentally validated miRNA:gene pairs found among 31 miRNAs and 62 genes indicative for CVD using multiMiR package. Table S12: Top 10\%-predicted miRNA:gene pairs found among 31 miRNAs and 62 genes indicative for CVD using multiMiR package.

Author Contributions: Conceptualization, A.B.-K., M.F. and J.K.; methodology, A.B.-K., D.P.Z., K.P.R., L.K., M.F. and J.K.; software, D.P.Z., L.K. and J.B.; validation, A.B.-K., D.P.Z., K.P.R. and M.F.; formal analysis, A.B.-K., D.P.Z., K.P.R., P.C. and J.B.; investigation, D.P.Z., K.P.R. and P.K.; resources, A.B.-K., A.S., M.F. and J.K.; data curation, D.P.Z., K.P.R. and A.S.; writing—original draft preparation, D.P.Z. and K.P.R.; writing—review and editing, A.B.-K., D.G. and M.F.; visualization, D.P.Z.; supervision, A.B.-K., A.S., M.F. and J.K.; project administration, A.B-K., A.S. and T.Z.; funding acquisition, A.B-K. and J.K. All authors have read and agreed to the published version of the manuscript.

Funding: This research and APC were funded by Statutory Funds of the Medical University of Lublin (number DS43 to A.B.-K.), provided by the Polish Ministry of Science and Higher Education for Medical University of Lublin, Poland.

Acknowledgments: The research was performed using the equipment purchased within the Project "The equipment of innovative laboratories doing research on new medicines used in the therapy of civilization and neoplastic diseases" within the Operational Program Development of Eastern Poland 2007-2013, Priority Axis I Modern Economy, Operations I.3 Innovation Promotion. 
Conflicts of Interest: A.B.-K., D.P.Z., K.P.R., A.S., P.K., T.Z., M.F. and J.K. are co-authors of patent application "Application of microRNA markers for diagnosis of chronic venous disease and method of chronic venous disease diagnosis" No. P.433137, submitted to Polish Patent Office.

Data Availability: All datasets generated for this study can be found in the FigShare repository https://doi.org/10. 6084/m9.figshare.12033738.v1.

\section{References}

1. Rabe, E.; Guex, J.J.; Puskas, A.; Scuderi, A.; Fernandez Quesada, F. VCP Coordinators. Epidemiology of chronic venous disorders in geographically diverse populations: Results from the Vein Consult Program. Int. Angiol. 2012, 31, 105-115. [PubMed]

2. Vuylsteke, M.E.; Colman, R.; Thomis, S.; Guillaume, G.; Van Quickenborne, D.; Staelens, I. An epidemiological survey of venous disease among general practitioner attendees in different geographical regions on the globe: The final results of the Vein Consult Program. Angiology 2018, 69, 779-785. [CrossRef] [PubMed]

3. Eklof, B.; Perrin, M.; Delis, K.; Rutherford, R. Updated terminology of chronic venous disorders: The vein term transatlantic interdisciplinary consensus document. J. Vasc. Surg. 2009, 49, 498-501. [CrossRef] [PubMed]

4. Rabe, E.; Pannier, F. Clinical, aetiological, anatomical and pathological classification (CEAP): Gold standard and limits. Phlebology 2012, 27 (Suppl. 1), 114-118. [CrossRef]

5. Eberhardt, R.T.; Raffetto, J.D. Chronic venous insufficiency. Circulation 2014, 130, 333-346. [CrossRef]

6. Lurie, F.; Passman, M.; Meisner, M.; Dalsing, M.; Masuda, E.; Welch, H.; Bush, R.L.; Blebea, J.; Carpentier, P.H.; De Maeseneer, M.; et al. CEAP classification system and reporting standard, revision 2020. J. Vasc. Surg. Venous Lymphat. Disord. 2020, 8, 342-352. [CrossRef]

7. Bergan, J.J.; Schmid-Schönbein, G.W.; Smith, P.D.; Nicolaides, A.N.; Boisseau, M.R.; Eklof, B. Chronic venous disease. N. Engl. J. Med. 2006, 355, 488-498. [CrossRef]

8. Meissner, M.H.; Moneta, G.; Burnand, K.; Gloviczki, P.; Lohr, J.M.; Lurie, F.; Mattos, M.A.; McLafferty, R.B.; Mozes, G.; Rutherford, R.B.; et al. The hemodynamics and diagnosis of venous disease. J. Vasc. Surg. 2007, 46 (Suppl. S), 4S-24S. [CrossRef]

9. Ligi, D.; Croce, L.; Mannello, F. Chronic venous disorders: The dangerous, the good, and the diverse. Int. J. Mol. Sci. 2018, 19, 2544. [CrossRef]

10. Bergan, J. Molecular mechanisms in chronic venous insufficiency. Ann. Vasc. Surg. 2007, 21, $260-266$. [CrossRef]

11. Pocock, E.S.; Alsaigh, T.; Mazor, R.; Schmid-Schönbein, G.W. Cellular and molecular basis of venous insufficiency. Vasc. Cell 2014, 6, 24. [CrossRef] [PubMed]

12. Mansilha, A.; Sousa, J. Pathophysiological mechanisms of chronic venous disease and implications for venoactive drug therapy. Int. J. Mol. Sci. 2018, 19, 1669. [CrossRef] [PubMed]

13. Serralheiro, P.; Novais, A.; Cairrão, E.; Maia, C.; Costa Almeida, C.M.; Verde, I. Variability of MMP/TIMP and TGF- $\beta 1$ receptors throughout the clinical progression of chronic venous disease. Int. J. Mol. Sci. 2018, 19, 6. [CrossRef] [PubMed]

14. Tracz, E.; Zamojska, E.; Modrzejewski, A.; Zaborski, D.; Grzesiak, W. Quality of life in patients with venous stasis ulcers and others with advanced venous insufficiency. Holist Nurs. Pract. 2015, 29, 96-102. [CrossRef]

15. Soydan, E.; Yilmaz, E.; Baydur, H. Effect of socio-demographic characteristics and clinical findings on the quality of life of patients with chronic venous insufficiency. Vascular 2017, 25, 382-389. [CrossRef]

16. Wittens, C.; Davies, A.H.; Bækgaard, N.; Broholm, R.; Cavezzi, A.; Chastanet, S.; de Wolf, M.; Eggen, C.; Giannoukas, A.; Gohel, M.; et al. Editor's Choice-Management of chronic venous disease: Clinical practice guidelines of the European Society for Vascular Surgery (ESVS). Eur. J. Vasc. Endovasc. Surg. 2015, 49, 678-737. [CrossRef]

17. Attaran, R.R. Latest innovations in the treatment of venous disease. J. Clin. Med. 2018, 7, 77. [CrossRef]

18. Ghildiyal, M.; Zamore, P.D. Small silencing RNAs: An expanding universe. Nat. Rev. Genet. 2009, 10, 94-108. [CrossRef]

19. Kim, D.; Sung, Y.M.; Park, J.; Kim, S.; Kim, J.; Park, J.; Ha, H.; Bae, J.Y.; Kim, S.; Baek, D. General rules for functional microRNA targeting. Nat. Genet. 2016, 48, 1517-1526. [CrossRef] 
20. Guo, H.; Ingolia, N.T.; Weissman, J.S.; Bartel, D.P. Mammalian microRNAs predominantly act to decrease target mRNA levels. Nature 2010, 466, 835-840. [CrossRef]

21. Mukherji, S.; Ebert, M.S.; Zheng, G.X.; Tsang, J.S.; Sharp, P.A.; van Oudenaarden, A. MicroRNAs can generate thresholds in target gene expression. Nat. Genet. 2011, 43, 854-859. [CrossRef] [PubMed]

22. Ohtsuka, M.; Ling, H.; Doki, Y.; Mori, M.; Calin, G.A. MicroRNA Processing and Human Cancer. J. Clin. Med. 2015, 4, 1651-1667. [CrossRef] [PubMed]

23. Rupaimoole, R.; Slack, F.J. MicroRNA therapeutics: Towards a new era for the management of cancer and other diseases. Nat. Rev. Drug Discov. 2017, 16, 203-222. [CrossRef] [PubMed]

24. Inamura, K.; Ishikawa, Y. MicroRNA in lung cancer: novel biomarkers and potential tools for treatment. J. Clin. Med. 2016, 5, 36. [CrossRef] [PubMed]

25. Qin, S.; Zhang, C. MicroRNAs in vascular disease. J. Cardiovasc. Pharmacol. 2011, 57, 8-12. [CrossRef]

26. Fernández-Hernando, C.; Suárez, Y. MicroRNAs in endothelial cell homeostasis and vascular disease. Curr. Opin. Hematol. 2018, 25, 227-236. [CrossRef]

27. Schulte, C.; Karakas, M.; Zeller, T. MicroRNAs in cardiovascular disease-Clinical application. Clin. Chem. Lab. Med. 2017, 55, 687-704. [CrossRef]

28. Feinberg, M.W.; Moore, K.J. MicroRNA regulation of atherosclerosis. Circ. Res. 2016, 118, 703-720. [CrossRef]

29. Zhou, S.S.; Jin, J.P.; Wang, J.Q.; Zhang, Z.G.; Freedman, J.H.; Zheng, Y.; Cai, L. miRNAS in cardiovascular diseases: Potential biomarkers, therapeutic targets and challenges. Acta Pharmacol. Sin. 2018, 39, 1073-1084. [CrossRef]

30. Bogucka-Kocka, A.; Zalewski, D.P.; Ruszel, K.P.; Stępniewski, A.; Gałkowski, D.; Bogucki, J.; Komsta, Ł.; Kołodziej, P.; Zubilewicz, T.; Feldo, M.; et al. Dysregulation of microRNA regulatory network in lower extremities arterial disease. Front. Genet. 2019, 10, 1200. [CrossRef]

31. Morelli, V.M.; Brækkan, S.K.; Hansen, J.-B. Role of microRNAs in venous thromboembolism. Int. J. Mol. Sci. 2020, 21, 2602. [CrossRef] [PubMed]

32. Markovic, J.N.; Shortell, C.K. Genomics of varicose veins and chronic venous insufficiency. Semin. Vasc. Surg. 2013, 26, 2-13. [CrossRef] [PubMed]

33. Cui, C.; Liu, G.; Huang, Y.; Lu, X.; Lu, M.; Huang, X.; Li, W.; Jiang, M. MicroRNA profiling in great saphenous vein tissues of patients with chronic venous insufficiency. Tohoku J. Exp. Med. 2012, 228, 341-350. [CrossRef] [PubMed]

34. Pastar, I.; Khan, A.A.; Stojadinovic, O.; Lebrun, E.A.; Medina, M.C.; Brem, H.; Kirsner, R.S.; Jimenez, J.J.; Leslie, C.; Tomic-Canic, M. Induction of specific microRNAs inhibits cutaneous wound healing. J. Biol. Chem. 2012, 287, 29324-29335. [CrossRef]

35. Jin, Y.; Xu, G.; Huang, J.; Zhou, D.; Huang, X.; Shen, L. Analysis of the association between an insertion/deletion polymorphism within the $3^{\prime}$ untranslated region of COL1A2 and chronic venous insufficiency. Ann. Vasc. Surg. 2013, 27, 959-963. [CrossRef]

36. Love, M.I.; Huber, W.; Anders, S. Moderated estimation of fold change and dispersion for RNA-seq data with DESeq2. Genome Biol. 2014, 15, 550. [CrossRef]

37. Centner, V.; Massart, D.L.; de Noord, O.E.; de Jong, S.; Vandeginste, B.M.; Sterna, C. Elimination of uninformative variables for multivariate calibration. Anal. Chem. 1996, 68, 3851-3858. [CrossRef]

38. Mehmood, T.; Hovde, K.H.; Liland Snipen, L.; Sæbø, S. A review of variable selection methods in Partial Least Squares Regression. Chemometr. Intell. Lab. Syst. 2012, 118, 62-69. [CrossRef]

39. Chen, H.; Boutros, P.C. VennDiagram: A package for the generation of highly-customizable Venn and Euler diagrams in R. BMC Bioinform. 2011, 12, 35. [CrossRef]

40. Ligges, U.; Mächler, M. Scatterplot3d-An R package for visualizing multivariate data. J. Stat. Softw. 2003, 8, 1-20. [CrossRef]

41. Robin, X.; Turck, N.; Hainard, A.; Tiberti, N.; Lisacek, F.; Sanchez, J.C.; Müller, M. pROC: An open-source package for R and $\mathrm{S}+$ to analyze and compare ROC curves. BMC Bioinform. 2011, 12, 77. [CrossRef] [PubMed]

42. Finotello, F.; Mayer, C.; Plattner, C.; Laschober, G.; Rieder, D.; Hackl, H.; Krogsdam, A.; Loncova, Z.; Posch, W.; Wilflingseder, D.; et al. Molecular and pharmacological modulators of the tumor immune contexture revealed by deconvolution of RNA-seq data. Genome Med. 2019, 11, 34. [CrossRef] [PubMed]

43. Becht, E.; Giraldo, N.A.; Lacroix, L.; Buttard, B.; Elarouci, N.; Petitprez, F.; Selves, J.; Laurent-Puig, P.; Sautès-Fridman, C.; Fridman, W.H.; et al. Estimating the population abundance of tissue-infiltrating immune and stromal cell populations using gene expression. Genome Biol. 2016, 17, 218. [CrossRef] 
44. Sturm, G.; Finotello, F.; Petitprez, F.; Zhang, J.D.; Baumbach, J.; Fridman, W.H.; List, M.; Aneichyk, T. Comprehensive evaluation of transcriptome-based cell-type quantification methods for immuno-oncology. Bioinformatics 2019, 35, i436-i445. [CrossRef] [PubMed]

45. Ru, Y.; Kechris, K.J.; Tabakoff, B.; Hoffman, P.; Radcliffe, R.A.; Bowler, R.; Mahaffey, S.; Rossi, S.; Calin, G.A.; Bemis, L.; et al. The multiMiR R package and database: Integration of microRNA-target interactions along with their disease and drug associations. Nucleic Acids Res. 2014, 42, e133. [CrossRef] [PubMed]

46. Shannon, P.; Markiel, A.; Ozier, O.; Baliga, N.S.; Wang, J.T.; Ramage, D.; Amin, N.; Schwikowski, B.; Ideker, T. Cytoscape: A software environment for integrated models of biomolecular interaction networks. Genome Res. 2003, 13, 2498-2504. [CrossRef]

47. Huang, D.W.; Sherman, B.T.; Lempicki, R.A. Systematic and integrative analysis of large gene lists using DAVID Bioinformatics Resources. Nat. Protoc. 2009, 4, 44-57. [CrossRef]

48. Huang, D.W.; Sherman, B.T.; Lempicki, R.A. Bioinformatics enrichment tools: Paths toward the comprehensive functional analysis of large gene lists. Nucleic Acids Res. 2009, 37, 1-13. [CrossRef]

49. Gloviczki, P.; Comerota, A.J.; Dalsing, M.C.; Eklof, B.G.; Gillespie, D.L.; Gloviczki, M.L.; Lohr, J.M.; McLafferty, R.B.; Meissner, M.H.; Murad, M.H.; et al. The care of patients with varicose veins and associated chronic venous diseases: Clinical practice guidelines of the Society for Vascular Surgery and the American Venous Forum. J. Vasc. Surg. 2011, 53 (Suppl. 5), 2S-48S. [CrossRef]

50. Han, H.; Qu, G.; Han, C.; Wang, Y.; Sun, T.; Li, F.; Wang, J.; Luo, S. MiR-34a, miR-21 and miR-23a as potential biomarkers for coronary artery disease: A pilot microarray study and confirmation in a 32 patient cohort. Exp. Mol. Med. 2015, 47, e138. [CrossRef]

51. Raitoharju, E.; Lyytikäinen, L.P.; Levula, M.; Oksala, N.; Mennander, A.; Tarkka, M.; Klopp, N.; Illig, T.; Kähönen, M.; Karhune, P.J.; et al. miR-21, miR-210, miR-34a, and miR-146a/b are up-regulated in human atherosclerotic plaques in the Tampere Vascular Study. Atherosclerosis 2011, 219, 211-217. [CrossRef] [PubMed]

52. Hernández-Walias, F.J.; Vázquez, E.; Pacheco, Y.; Rodríguez-Fernández, J.M.; Pérez-Elías, M.J.; Dronda, F.; Casado, J.L.; Moreno, A.; Hermida, J.M.; Quereda, C.; et al. Risk, diagnostic and predictor factors for classical hodgkin lymphoma in HIV-1-infected individuals: Role of plasma exosome-derived miR-20a and miR-21. J. Clin. Med. 2020, 9, 760. [CrossRef]

53. Irimie-Aghiorghiesei, A.I.; Pop-Bica, C.; Pintea, S.; Braicu, C.; Cojocneanu, R.; Zimța, A.-A.; Gulei, D.; Slabý, O.; Berindan-Neagoe, I. Prognostic Value of MiR-21: An updated meta-analysis in Head and Neck Squamous Cell Carcinoma (HNSCC). J. Clin. Med. 2019, 8, 2041. [CrossRef] [PubMed]

54. Shi, J. Considering exosomal miR-21 as a biomarker for cancer. J. Clin. Med. 2016, 5, 42. [CrossRef] [PubMed]

55. Ulivi, P.; Petracci, E.; Marisi, G.; Baglivo, S.; Chiari, R.; Billi, M.; Canale, M.; Pasini, L.; Racanicchi, S.; Vagheggini, A.; et al. Prognostic role of circulating miRNAs in early-stage non-small cell lung cancer. J. Clin. Med. 2019, 8, 131. [CrossRef]

56. Saito, Y.; Nakaoka, T.; Saito, H. microRNA-34a as a therapeutic agent against human cancer. J. Clin. Med. 2015, 4, 1951-1959. [CrossRef]

57. Cario-Toumaniantz, C.; Boularan, C.; Schurgers, L.J.; Heymann, M.F.; Le Cunff, M.; Léger, J.; Loirand, G.; Pacaud, P. Identification of differentially expressed genes in human varicose veins: Involvement of matrix gla protein in extracellular matrix remodeling. J. Vasc. Res. 2007, 44, 444-459. [CrossRef]

58. Charles, C.A.; Tomic-Canic, M.; Vincek, V.; Nassiri, M.; Stojadinovic, O.; Eaglstein, W.H.; Kirsner, R.S. A gene signature of nonhealing venous ulcers: Potential diagnostic markers. J. Am. Acad Dermatol. 2008, 59, 758-771. [CrossRef]

59. Chang, M.Y.; Chiang, P.T.; Chung, Y.C.; Ho, S.Y.; Lin, S.D.; Lin, S.R.; Neoh, C.A. Apoptosis and angiogenesis in varicose veins using gene expression profiling. Fooyin J. Health Sci. 2009, 1, 85-91. [CrossRef]

60. Hsieh, C.S.; Tsai, C.T.; Chen, Y.H.; Chang, S.N.; Hwang, J.J.; Chuang, E.Y.; Wu, I.H. Global expression profiling identifies a novel hyaluronan synthases 2 gene in the pathogenesis of lower extremity varicose veins. J. Clin. Med. 2018, 7, 537. [CrossRef]

61. Dbouk, H.A.; Weil, L.M.; Perera, G.K.; Dellinger, M.T.; Pearson, G.; Brekken, R.A.; Cobb, M.H. Actions of the protein kinase WNK1 on endothelial cells are differentially mediated by its substrate kinases OSR1 and SPAK. Proc. Natl. Acad. Sci. USA 2014, 111, 15999-16004. [CrossRef]

62. Sun, X.; Sit, A.; Feinberg, M.W. Role of miR-181 family in regulating vascular inflammation and immunity. Trends Cardiovasc. Med. 2014, 24, 105-112. [CrossRef] [PubMed] 
63. Xie, W.; Li, M.; Xu, N.; Lv, Q.; Huang, N.; He, J.; Zhang, Y. MiR-181a regulates inflammation responses in monocytes and macrophages. PLoS ONE 2013, 8, e58639. [CrossRef] [PubMed]

64. Du, X.J.; Lu, J.M.; Sha, Y. MiR-181a inhibits vascular inflammation induced by ox-LDL via targeting TLR4 in human macrophages. J. Cell Physiol. 2018, 233, 6996-7003. [CrossRef] [PubMed]

65. Zhu, J.; Yao, K.; Wang, Q.; Guo, J.; Shi, H.; Ma, L.; Liu, H.; Gao, W.; Zou, Y.; Ge, J. Circulating miR-181a as a potential novel biomarker for diagnosis of acute myocardial infarction. Cell Physiol. Biochem. 2016, 40, 1591-1602. [CrossRef]

66. Yentrapalli, R.; Azimzadeh, O.; Kraemer, A.; Malinowsky, K.; Sarioglu, H.; Becker, K.F.; Atkinson, M.J.; Moertl, S.; Tapio, S. Quantitative and integrated proteome and microRNA analysis of endothelial replicative senescence. J. Proteomics. 2015, 126, 12-23. [CrossRef]

67. Ayaz, L.; Dinç, E. Evaluation of microRNA responses in ARPE-19 cells against the oxidative stress. Cutan. Ocul. Toxicol. 2018, 37, 121-126. [CrossRef]

68. Zhou, J.; He, Z.; Guo, L.; Zeng, J.; Liang, P.; Ren, L.; Zhang, M.; Zhang, P.; Huang, X. MiR-128-3p directly targets VEGFC/VEGFR3 to modulate the proliferation of lymphatic endothelial cells through $\mathrm{Ca}(2+)$ signaling. Int. J. Biochem. Cell Biol. 2018, 102, 51-58. [CrossRef]

69. Xia, Z.; Meng, F.; Liu, Y.; Fang, Y.; Wu, X.; Zhang, C.; Liu, D.; Li, G. Decreased MiR-128-3p alleviates the progression of rheumatoid arthritis by up-regulating the expression of TNFAIP3. Biosci. Rep. 2018, 38, BSR20180540. [CrossRef]

70. Liu, S.; Zhu, J.; Jiang, T.; Zhong, Y.; Tie, Y.; Wu, Y.; Zheng, X.; Jin, Y.; Fu, H. Identification of lncRNA MEG3 binding protein using MS2-Tagged RNA affinity purification and mass spectrometry. Appl. Biochem. Biotechnol. 2015, 176, 1834-1845. [CrossRef]

71. Zhang, X.; Zhou, Y.; Mehta, K.R.; Danila, D.C.; Scolavino, S.; Johnson, S.R.; Klibanski, A. A pituitary-derived MEG3 isoform functions as a growth suppressor in tumor cells. J. Clin. Endocrinol. Metab. 2003, 88, 5119-5126. [CrossRef]

72. Zhou, Y.; Zhong, Y.; Wang, Y.; Zhang, X.; Batista, D.L.; Gejman, R.; Ansell, P.J.; Zhao, J.; Weng, C.; Klibanski, A. Activation of p53 by MEG3 non-coding RNA. J. Biol. Chem. 2007, 282, 24731-24742. [CrossRef]

73. Gong, L.; Xu, H.; Chang, H.; Tong, Y.; Zhang, T.; Guo, G. Knockdown of long non-coding RNA MEG3 protects H9c2 cells from hypoxia-induced injury by targeting microRNA-183. J. Cell Biochem. 2018, 119, 1429-1440. [CrossRef] [PubMed]

74. Zhang, Y.J.; Zheng, H.Q.; Chen, B.Y.; Sun, L.; Ma, M.M.; Wang, G.L.; Guan, Y.Y. WNK1 is required for proliferation induced by hypotonic challenge in rat vascular smooth muscle cells. Acta Pharmacol. Sin. 2018, 39, 35-47. [CrossRef] [PubMed]

75. Wang, L.; Xu, M.; Lu, P.; Zhou, F. MicroRNA-769 is downregulated in colorectal cancer and inhibits cancer progression by directly targeting cyclin-dependent kinase 1 . Onco Targets Ther. 2018, 11,9013-9025. [CrossRef] [PubMed]

76. Yang, Z.; He, J.; Gao, P.; Niu, Y.; Zhang, J.; Wang, L.; Liu, M.; Wei, X.; Liu, C.; Zhang, C.; et al. MiR-769-5p suppressed cell proliferation, migration and invasion by targeting TGFBR1 in non-small cell lung carcinoma. Oncotarget 2017, 8, 113558-113570. [CrossRef]

77. Busch, A.; Busch, M.; Scholz, C.J.; Kellersmann, R.; Otto, C.; Chernogubova, E.; Maegdefessel, L.; Zernecke, A.; Lorenz, U. Aneurysm miRNA Signature Differs, Depending on Disease Localization and Morphology. Int. J. Mol. Sci. 2016, 17, 81. [CrossRef]

78. Xu, X.; Ha, C.H.; Wong, C.; Wang, W.; Hausser, A.; Pfizenmaier, K.; Olson, E.N.; McKinsey, T.A.; Jin, Z.G. Angiotensin II stimulates protein kinase D-dependent histone deacetylase 5 phosphorylation and nuclear export leading to vascular smooth muscle cell hypertrophy. Arterioscler. Thromb. Vasc. Biol. 2007, 27, 2355-2362. [CrossRef]

79. Wang, M.; Ji, Y.; Cai, S.; Ding, W. MiR-206 suppresses the progression of coronary artery disease by modulating Vascular Endothelial Growth Factor (VEGF) Expression. Med. Sci. Monit. 2016, 22, 5011-5020. [CrossRef]

80. Stahlhut, C.; Suárez, Y.; Lu, J.; Mishima, Y.; Giraldez, A.J. MiR-1 and miR-206 regulate angiogenesis by modulating VegfA expression in zebrafish. Development 2012, 139, 4356-4364. [CrossRef]

81. Lai, J.G.; Tsai, S.M.; Tu, H.C.; Chen, W.C.; Kou, F.J.; Lu, J.W.; Wang, H.D.; Huang, C.L.; Yuh, C.H. Zebrafish WNK lysine deficient protein kinase 1 (wnk1) affects angiogenesis associated with VEGF signaling. PLoS ONE 2014, 9, e106129. [CrossRef] [PubMed] 
82. Pan, W.; Pham, V.N.; Stratman, A.N.; Castranova, D.; Kamei, M.; Kidd, K.R.; Lo, B.D.; Shaw, K.M.; Torres-Vazquez, J.; Mikelis, C.M.; et al. CDP-diacylglycerol synthetase-controlled phosphoinositide availability limits VEGFA signaling and vascular morphogenesis. Blood 2012, 120, 489-498. [CrossRef] [PubMed]

83. Brugarolas, J.B.; Vazquez, F.; Reddy, A.; Sellers, W.R.; Kaelin, W.G., Jr. TSC2 regulates VEGF through mTOR-dependent and -independent pathways. Cancer Cell 2003, 4, 147-158. [CrossRef]

84. Deroanne, C.F.; Bonjean, K.; Servotte, S.; Devy, L.; Colige, A.; Clausse, N.; Blacher, S.; Verdin, E.; Foidart, J.M.; Nusgens, B.V.; et al. Histone deacetylases inhibitors as anti-angiogenic agents altering vascular endothelial growth factor signaling. Oncogene 2002, 21, 427-436. [CrossRef]

85. Urbich, C.; Rössig, L.; Kaluza, D.; Potente, M.; Boeckel, J.N.; Knau, A.; Diehl, F.; Geng, J.G.; Hofmann, W.K.; Zeiher, A.M.; et al. HDAC5 is a repressor of angiogenesis and determines the angiogenic gene expression pattern of endothelial cells. Blood 2009, 113, 5669-5679. [CrossRef]

86. Wang, X.T.; Wu, X.D.; Lu, Y.X.; Sun, Y.H.; Zhu, H.H.; Liang, J.B.; He, W.K.; Zeng, Z.Y.; Li, L. Potential involvement of mir-30e-3p in myocardial injury induced by coronary microembolization via autophagy activation. Cell Physiol. Biochem. 2017, 44, 1995-2004. [CrossRef]

87. Kim, S.H.; Kim, G.J.; Umemura, T.; Lee, S.G.; Cho, K.J. Aberrant expression of plasma microRNA-33a in an atherosclerosis-risk group. Mol. Biol. Rep. 2017, 44, 79-88. [CrossRef]

88. Dong, J.; Liang, Y.Z.; Zhang, J.; Wu, L.J.; Wang, S.; Hua, Q.; Yan, Y.X. Potential role of lipometabolism-related micrornas in peripheral blood mononuclear cells as biomarkers for coronary artery disease. J. Atheroscler. Thromb. 2017, 24, 430-441. [CrossRef]

89. Deng, X.; Qin, S.; Chen, Y.; Liu, H.Y.; Yuan, E.; Deng, H.; Liu, S.M. B-RCA revealed circulating miR-33a/b associates with serum cholesterol in type 2 diabetes patients at high risk of ASCVD. Diabetes Res. Clin. Pract. 2018, 140, 191-199. [CrossRef]

90. Siedlecki-Wullich, D.; Català-Solsona, J.; Fábregas, C.; Hernández, I.; Clarimon, J.; Lleó, A.; Boada, M.; Saura, C.A.; Rodríguez-Álvarez, J.; Miñano-Molina, A.J. Altered microRNAs related to synaptic function as potential plasma biomarkers for Alzheimer's disease. Alzheimers Res. Ther. 2019, 11, 46. [CrossRef]

91. Ma, J.; Shang, S.; Wang, J.; Zhang, T.; Nie, F.; Song, X.; Zhao, H.; Zhu, C.; Zhang, R.; Hao, D. Identification of miR-22-3p, miR-92a-3p, and miR-137 in peripheral blood as biomarker for schizophrenia. Psychiatry Res. 2018, 265, 70-76. [CrossRef] [PubMed]

92. Mei, Q.; Liu, J.; Liu, Y.; Li, C.; Wang, H.; Li, H.; Chen, X.; Lan, X. Expression of proline-rich coiled-coil 2B protein in developing rat brains. Neurosci. Lett. 2013, 557 Pt B, 171-176. [CrossRef]

93. Fujii, T.; Shimada, K.; Nakai, T.; Ohbayashi, C. MicroRNAs in smoking-related carcinogenesis: Biomarkers, functions, and therapy. J. Clin. Med. 2018, 7, 98. [CrossRef] [PubMed] 